\title{
Developing Formulas for Quick Calculation of Polyhedron Volume in Spatial Geometry: Application to Vietnam
}

\author{
Bui Anh Tuan ${ }^{1}$, Michael McAleer ${ }^{2-6}$, Nguyen Thi Tieu Dang ${ }^{7}$ and Kim-Hung Pho ${ }^{8,}$ \\ ${ }^{1}$ Department of Mathematics Education, Teachers College, Can Tho University, Vietnam \\ ${ }^{2}$ Department of Finance, Asia University, Taiwan \\ ${ }^{3}$ Discipline of Business Analytics, University of Sydney Business School, Australia \\ ${ }^{4}$ Econometric Institute, Erasmus School of Economics, Erasmus University Rotterdam, The Netherlands \\ ${ }^{5}$ Department of Economic Analysis and ICAE, Complutense University of Madrid, Spain \\ ${ }^{6}$ Institute of Advanced Sciences, Yokohama National University, Japan \\ ${ }^{7}$ Department of Mathematics Education, Teachers College, Can Tho University, Vietnam \\ ${ }^{8}$ Fractional Calculus, Optimization and Algebra Research Group, Faculty of Mathematics and Statistics, Ton \\ Duc Thang University, Ho Chi Minh City, Vietnam
}

\begin{abstract}
In the age of globalization, an effective leadership skill is the ability for quick calculation of work-related problems. From an economic perspective, fast computation often provides a competitive advantage in business, where speed, efficiency and accuracy are required. Quick calculation techniques are a central problem in modern mathematics because it shortens the time for solving technical problems. The purpose of the paper is to provide an explanation that will lead to a quick solution to a volume problem. Specifically, some convenient formulas are provided for quick calculation of the volume of the common polyhedron, together with a number of multiple-choice questions with IATA software to practice. Based on the evaluation results, reliable multiple-choice questions are used for an empirical study in Can Tho City, Vietnam on the effectiveness of the formulas for quick calculation of the polyhedron volume in spatial geometry. Statistical analysis shows that quick formulas help students to complete lessons at a higher rate, thereby contributing to improvements in the effectiveness of teaching geometry, especially the volume of the Polyhedron.
\end{abstract}

Keywords: Leadership, Knowledge Production, Polyhedron, Volume, Spatial Geometry, Quick calculation.

\section{INTRODUCTION}

In the age of globalization, an effective leadership skill is the ability for quick calculation of work-related problems. From an economic perspective, fast computation often provides a competitive advantage in business, where speed, efficiency and accuracy are not only useful but also essential. Quick calculation techniques are a central problem in modern mathematics because it shortens the time for solving technical problems.

As an example of an interesting problem in spatial geometry, consider a company that produces a monolithic wooden souvenir in the shape of a pyramid, as shown below. Knowing the wood block with the bottom side is $6 \mathrm{~cm}$ square, the side is $9 \mathrm{~cm}$ long. How many $\mathrm{cm}^{3}$ of wood are needed to complete this pyramid?

*Address correspondence to this author at the Fractional Calculus, Optimization and Algebra Research Group, Faculty of Mathematics and Statistics, Ton Duc Thang University, Ho Chi Minh City, Vietnam;

E-mail: phokimhung@tdtu.edu.vn

JEL: C63, D83

E-ISSN: 1929-7092/19

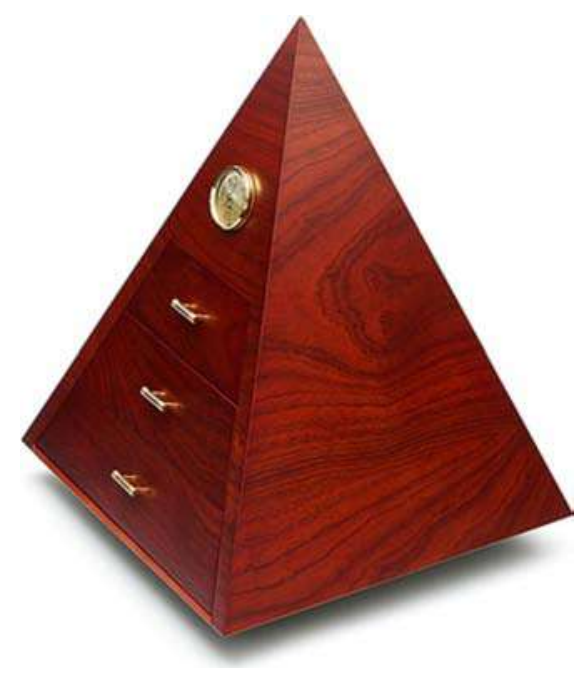

In order to address this problem, one needs to build a mathematical model, then use geometric formulas to calculate the solution. This work is quite simple for a mathematician, but it may not be quite as simple for others. The question to be addressed can be presented as: "How can a system of formulas be constructed for a quick calculation of polyhedron volume in spatial geometry in order to solve problems in real life?"

Returning to the initial problem, call the bottom and side edges of the wooden pyramid $a$ and $b$,

(c) 2019 Lifescience Global 
respectively. Then it is possible to formulate the volume of wood blocks as:

$V=\frac{a^{2} \sqrt{4 b^{2}-2 a^{2}}}{6}$

With $a=6 \mathrm{~cm}$ and $b=9 \mathrm{~cm}$, using the formula leads to a quick calculation of the volume of wood.

From the illustration above, it can be seen that the construction of a quick calculation system of the pyramid volume has two benefits:

1. The time to calculate the volume of the pyramid problem will be faster;

2. The quick calculation formula can be programmed easily.

The procedure for establishing the formula can be considered as a type of knowledge production. According to Bribena (2019), the production of such knowledge is closely related to the process of globalization, with both offering important elements in the global commodity and commercial markets, where speedy computation can provide a competitive advantage. The process of producing knowledge also plays an essential role in categorizing global organizations in different systems.

From a different perspective, according to Sousa et al. (2019), to be a good leader in the industrial age 4.0 requires at least two qualities, namely good work and computational thinking. With the construction of quick calculation formulas, its use by skillful leaders will save time and improve work efficiency. Creating such a recipe also helps to train people to learn computational skills develop a specific mindset, thereby connecting the real world to its virtual counterpart.

From an educational perspective, mathematics is a very important and influential subject for admission to a university or college. Therefore, in order to obtain a high score in this subject, we need to have knowledge and understanding of mathematical concepts and essences. In particular, spatial geometry has the most obvious difference for algebra which is the theory of space, thus learners should study theories as well as theorems to be able to apply effectively.

In order to address the problems of spatial geometry, it is important to draw the right shape. Imagine how you can see the surface, not see the surface, from which one can know the shapes that we need to calculate it. But to be able to solve quickly to spatial geometry, it is crucial to practice this issue regularly, because only practice will increase the ability to think and apply theorems quickly. About spatial geometry, this issue is currently researched theoretically and practically by several scientists and has gained many attentions in the last two decades.

For instance, Bertoline (1991) presented to use 3D geometric models to teach spatial geometry concepts. Bako (2003) researched about different projecting methods in teaching spatial geometry. Laborde et al. (2006) introduced about teaching and learning geometry with technology. Kosa and Karakus, F. (2010) researched to dynamic geometry software Cabri $3 \mathrm{D}$ for teaching analytic geometry. Kurtulus and Uygan (2010) presented to the effects of Google Sketchup based geometry activities and projects on spatial visualization ability of student mathematics teachers. Moss et al. (2015) introduced about adapting Japanese Lesson Study to enhance the teaching and learning of geometry and spatial reasoning in early year classrooms: a case study. Peter (2012) introduced to critical thinking: Essence for teaching mathematics and mathematics problem solving skills.

In Vietnam, the renewal of the form of multiplechoice assessment of mathematics from National high school exams from 2017 shows progress in education reform. Since then, the renewal of teaching and learning methods to suit new exam forms has also been continuously set and achieved significant results. Nevertheless, to meet that requirement, students need a tremendous amount of knowledge to solve 50 multiple-choice questions within 90 minutes. In which questions about spatial geometry still occupy relatively large time of students. About multiple-choice questions, there are many scientists have studied and utilized it, for example, Lindberg et al. (2010) researched to new trends in gender and mathematics performance: a meta-analysis.

Torres et al. (2011) presented about improving Multiple-Choice Questions. Little et al. (2012) introduced to multiple-choice tests exonerated, at least of some charges: Fostering test-induced learning and avoiding test-induced forgetting. Lesage et al. (2013) researched about scoring methods for multiple choice assessment in higher education-ls it still a matter of number right scoring or negative marking? Lindner et al. (2014) presented about tracking the decision-making process in multiple-choice assessment: Evidence from eye movements. Azevedo (2015) introduced to e-assessment in mathematics courses with multiple-choice questions tests. 
Regarding the use of formulas in teaching mathematics, Chazan (2000) proved effective when applying the Algebraic formula system in High Schools. For quick manipulations, Wheatley (2016) has developed rapid drawing techniques applied in mathematics; Boslaugh (2012) provides a statistical view of fast citation tools on the desktop. In Geometry, Grabinski et al. (2002) also studied simple formulas related to straight lines. From studies and context of teaching mathematics in Vietnam, a question arises: "How to build formulas for quick calculation of polyhedron volumes in geometry for the teaching of mathematics?"

In order to provide an answer to this question, this paper first introduce to formulas to quickly calculate of polyhedron volume in spatial geometry based on the knowledge learned to help students solve multiplechoice questions quickly and effectively. This is the primary motivation for us to study this article. The rest of the paper is organized as follows. The literature review is presented in Section 2. We develop formulas to quickly calculate of polyhedron volume in spatial geometry in Section 3. In Section 4, we investigate to empirical analysis at Can Tho City to evaluate efficiently of the proposed formulas. Concluding remarks and inference will be provided in the last section.

\section{LITERATURE REVIEW}

\subsection{Pyramid}

Given convex polygons $A_{1} A_{2} \ldots A_{n}$ and a point $\mathrm{S}$ is outside the plane containing that polygon. Connecting $\mathrm{S}$ with vertices $A_{1}, A_{2}, \ldots, A_{n}$ to obtain $\mathrm{n}$ triangles: $S A_{1} A_{2}, S A_{2} A_{3}, \ldots, S A_{n} A_{1}$.

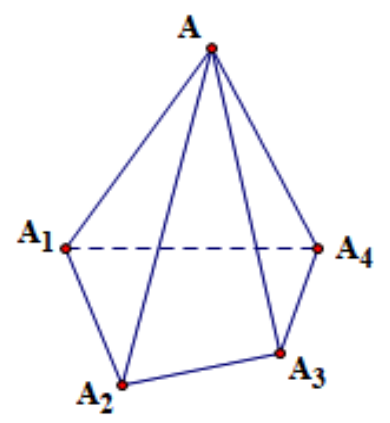

Shapes consists of $\mathrm{n}$ triangles and polygons $A_{1} A_{2} \ldots A_{n}$ is called a pyramid and it denoted by S. $A_{1} A_{2} \ldots A_{n}$.

\subsection{Equilateral Pyramid}

A pyramid is called an equilateral pyramid if its base is a regular polygon and its sides are equal. Sometimes we also define the following: A pyramid is called an equilateral pyramid if its base is a regular polygon and has the ending point of perpendicular lines that coincides with the center of the base polygon.

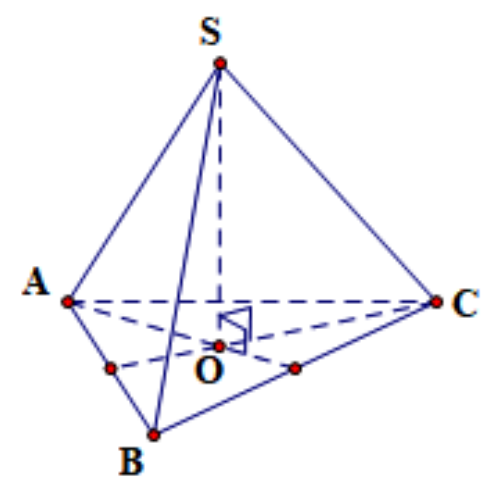

In an equilateral pyramid, it can be seen that, side edges and side surfaces create the base polygons of equal angles.

\subsection{Prisms}

Shapes is created by parallelograms $A_{1} A_{2} A_{2}^{\prime} A_{1}^{\prime}, A_{2} A_{3} A^{\prime}{ }_{3} A_{2}^{\prime}, \ldots, A_{n} A_{1} A_{1}^{\prime} A^{\prime}{ }_{n} \quad$ and two polygons $A_{1} A_{2} \ldots A_{n}, A_{1}^{\prime} A_{2}^{\prime}{ }_{2} \ldots A_{n}^{\prime}$ is called a prism, and it denoted by $A_{1} A_{2} \ldots A_{n} \cdot A_{1}^{\prime} A_{2}^{\prime}{ }_{2} \ldots A_{n}^{\prime}$.

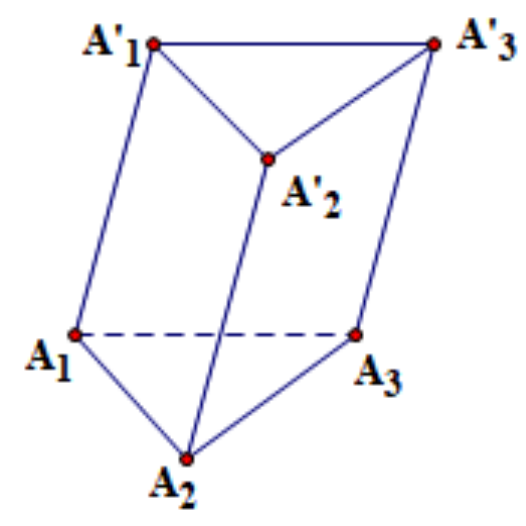

In a prism, it has been seen that, the side edges are equal, the side surfaces are parallelograms and two bottoms are equal polygons.

\subsection{Box Shape}

A box shape is a prism has the base is a parallelogram. 


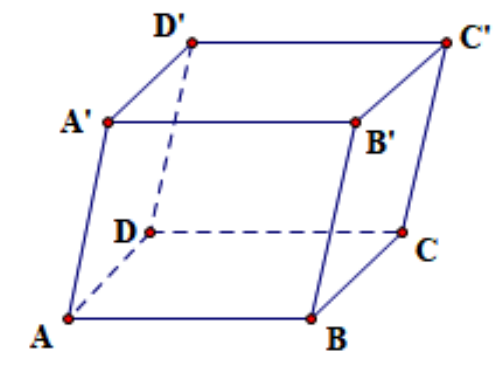

In a box shape, it can be seen that the side surfaces are parallelograms and the diagonal of the box shape intersects at the center of each line. We now introduce to formulas to quickly calculate of polyhedron volume in spatial geometry in the next section.

\section{DEVELOPING FORMULAS FOR QUICK CALCULATION OF POLYHEDRON VOLUME IN SPATIAL GEOMETRY}

According to Jones (2002), the demonstration of Mathematical properties is conducted in the following order: Explanation $\rightarrow$ Discovery $\rightarrow$ Intellectual challenge $\rightarrow$ Verification $\rightarrow$ Systematization.

This process has two major problems for learners: proof time and intelligence. To shorten the process, we demonstrate the general formulas of the volume of common polyhedron. In order to shorten the time, we have developed a recipe table for learners. The following presentation relates to the proof and formulation of the general formula.

\subsection{Volume of pyramid}

\subsubsection{Equilateral Triangular Pyramid}

Formula 1: Given an equilateral triangular pyramid $S . \overline{A B C}$ has a base $A B C$ is an equilateral triangle with its edges equal to $a$ and the side edge equals to $b$, then one has:

$V_{S . A B C}=\frac{a^{2} \sqrt{3 b^{2}-a^{2}}}{12}$

\section{$\underline{\text { Solution }}$}

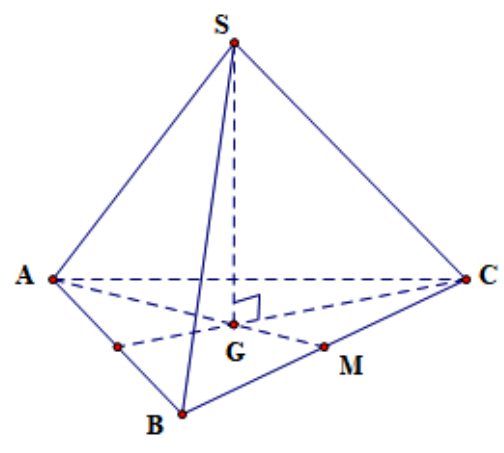

Let $G$ is a center of $\triangle A B C \Rightarrow S G \perp(A B C)$

It can be observed that $\triangle A B C$ is an equilateral triangular

$$
\Rightarrow A M=\frac{a \sqrt{3}}{2} \Rightarrow A G=\frac{a \sqrt{3}}{3}
$$
thus:

In addition, we have $\triangle S G A$ is a right triangle at $G$

$$
\begin{aligned}
& S G=\sqrt{S A^{2}-A G^{2}}=\sqrt{b^{2}-\frac{a^{2}}{3}}=\sqrt{\frac{3 b^{2}-a^{2}}{3}} \\
& \text { Hence, } V_{S . A B C}=\frac{1}{3} \cdot S G \cdot S_{\triangle A B C}=\frac{1}{3} \cdot \sqrt{\frac{3 b^{2}-a^{2}}{3}} \cdot \frac{a^{2} \sqrt{3}}{4}=\frac{a^{2} \sqrt{3 b^{2}-a^{2}}}{12} \\
& \text { In case of } a=b \Rightarrow V=\frac{a^{3} \sqrt{2}}{12} .
\end{aligned}
$$

Formula 2: Given an equilateral triangular pyramid $S . A B C$ has a base edge with length equal to a and the side surface created with the base plane is an angle is $\alpha$, then one has:

$V_{S . A B C}=\frac{a^{3} \tan \alpha}{24}$

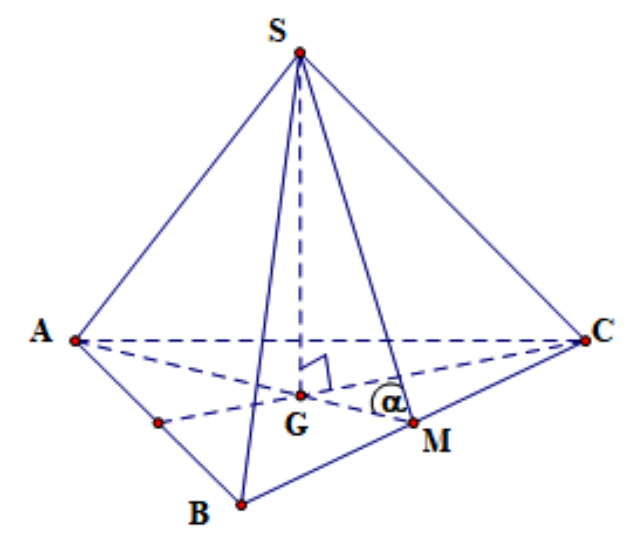

Solution

Let $G$ is a center of $\triangle A B C \Rightarrow S G \perp(A B C)$

Thus the angle of between $(S B C)$ and $(A B C)$ is $\widehat{S M G}=\alpha$

One has $\triangle A B C$ is an equilateral triangular

$\Rightarrow S_{\triangle A B C}=\frac{a^{2} \sqrt{3}}{4}$

Furthermore, we have $\triangle S M G$ is a right triangle at $G$, thereafter, we get: 
$S G=G M \cdot \tan \alpha=\frac{1}{3} A M \cdot \tan \alpha=\frac{a \sqrt{3} \cdot \tan \alpha}{6}$

and obtain $V_{S \cdot A B C}=\frac{1}{3} \cdot S G \cdot S_{\triangle A B C}=\frac{1}{3} \cdot \frac{a \sqrt{3} \cdot \tan \alpha}{6} \cdot \frac{a^{2} \sqrt{3}}{4}=\frac{a^{3} \tan \alpha}{24}$.

Formula 3: Given an equilateral triangular pyramid $S . A B C$ has a side edge with length equal to $\mathrm{b}$ and the side edge created with the base plane is an angle is $\beta$, then one has:

$$
V_{S . A B C}=\frac{\sqrt{3} b^{3} \sin \beta \cos ^{2} \beta}{4}
$$

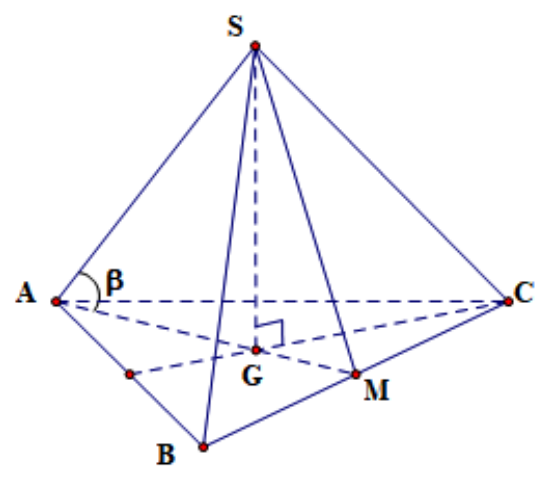

Solution

Let $G$ is a center of $\triangle A B C \Rightarrow S G \perp(A B C)$

$\triangle S G A$ is a right triangle at $G$, one has:

$S G=S A \cdot \sin \beta=b \cdot \sin \beta$

In addition, we have

$A G=S A \cdot \cos \beta \Rightarrow A M=\frac{3}{2} A G=\frac{3 b \cdot \cos \beta}{2}$

On the other hand, $\triangle A B C$ is an equilateral triangular, one has

$\Rightarrow A M=\frac{\sqrt{3}}{2} A B \Rightarrow A B=\frac{2}{\sqrt{3}} A M=\sqrt{3} b \cdot \cos \beta$

$\Rightarrow S_{\triangle A B C}=\frac{A B^{2} \sqrt{3}}{4}=(\sqrt{3} b \cdot \cos \beta)^{2} \cdot \frac{\sqrt{3}}{4}=\frac{3 \sqrt{3} b^{2} \cos ^{2} \beta}{4}$

Therefore, $V_{S . A B C}=\frac{1}{3} \cdot S G \cdot S_{\triangle A B C}=\frac{\sqrt{3} b^{3} \cdot \sin \beta \cos ^{2} \beta}{4}$.

Formula 4: Given an equilateral triangular pyramid $S . A B C$ has a base edge with length equal to a and the side edge created with the base plane is an angle is $\beta$, then one has :
$V_{S . A B C}=\frac{a^{3} \tan \beta}{12}$

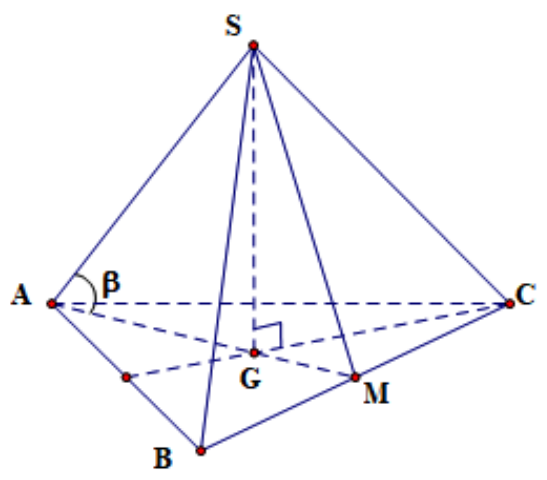

Solution

Let $G$ is a center of $\triangle A B C \Rightarrow S G \perp(A B C)$

It has been seen that, $\triangle A B C$ is an equilateral triangular

$\Rightarrow A M=\frac{\sqrt{3}}{2} A B=\frac{\sqrt{3}}{2} a$

$\triangle S G A$ is a right triangle at $G$, thereafter we get:

$S G=A G \cdot \tan \beta=\frac{2}{3} A M \cdot \tan \beta=\frac{\sqrt{3}}{3} a \cdot \tan \beta$

and obtain $\quad V_{S \cdot A B C}=\frac{1}{3} \cdot S G \cdot S_{\triangle A B C}=\frac{1}{3} \cdot \frac{\sqrt{3}}{3} a \cdot \tan \beta \cdot \frac{a^{2} \sqrt{3}}{4}$ $=\frac{a^{3} \cdot \tan \beta}{12}$.

Formula 5: Given an equilateral triangular pyramid $S . A B C$ has a base edge with length equal to a. Let $(P)$ is the plane passing through $A$ and parallel to $B C$, and perpendicular to the plane $(S B C)$, the angle between the plane $(\mathrm{P})$ and the base plane is $\alpha$, then one has:

$V_{S . A B C}=\frac{a^{3} \cot \alpha}{24}$

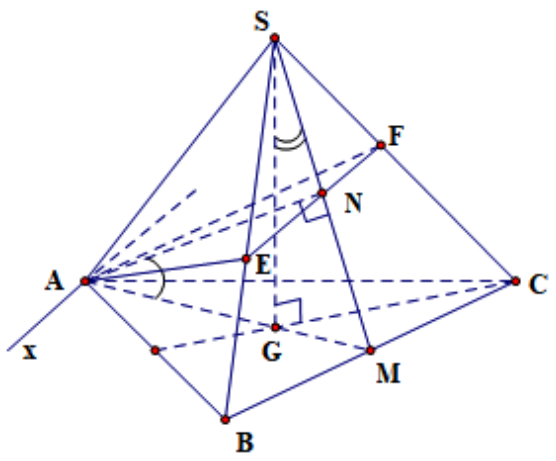




\section{Solution}

Let $G$ is a center of $\triangle A B C \Rightarrow S G \perp(A B C)$

It can be observed that, $\triangle A B C$ is an equilateral triangular

$\Rightarrow S_{\triangle A B C}=\frac{a^{2} \sqrt{3}}{4}$

We have $E F=(P) \cap(S B C) \Rightarrow E F / / B C$

$\Rightarrow(P) \cap(A B C)=A x$ with $A x / / E F / / B C$

Let $\mathrm{M}$ is the midpoint of $\mathrm{BC}$, and $N=S M \cap E F$

One has $\left\{\begin{array}{l}B C \perp A M \\ B C \perp S G\end{array} \Rightarrow B C \perp(S A M) \Rightarrow A N \perp B C \Rightarrow A N \perp A x\right.$

On the other hand, $\left\{\begin{array}{l}A M \perp B C \\ B C / / A x\end{array} \Rightarrow A M \perp A x\right.$ $\Rightarrow((P),(A B C))=\widehat{N A M}=\alpha$

In addition, we have $\widehat{G S M}=\widehat{N A M}=\alpha$ (supplementary with $\widehat{S M A}$ )

$\triangle S G A$ is a right triangle at $M$, thereafter we obtain:

$S G=G M \cdot \cot \alpha=\frac{1}{3} A M \cdot \cot \alpha=\frac{1}{3} \cdot \frac{a \sqrt{3}}{2} \cdot \cot \alpha=\frac{a \sqrt{3} \cdot \cot \alpha}{6}$

and get, $S_{S \cdot A B C}=\frac{1}{3} \cdot S G \cdot S_{\triangle A B C}=\frac{1}{3} \cdot \frac{a \sqrt{3} \cdot \cot \alpha}{6} \cdot \frac{a^{2} \sqrt{3}}{4}=\frac{a^{3} \cdot \cot \alpha}{24}$.

\subsubsection{Equilateral Quadrilateral Pyramid}

Formula 6: Given an equilateral quadrilateral pyramid $S . A B C D$ with its base $A B C D$ is a square with the length of side is a and its side edge with the length equal to $b$ :

$V_{S . A B C D}=\frac{a^{2} \sqrt{4 b^{2}-2 a^{2}}}{6}$

If an equilateral quadrilateral pyramid has all of sides with the length equal to a, one has:

$V_{S . A B C D}=\frac{a^{3} \sqrt{2}}{6}$

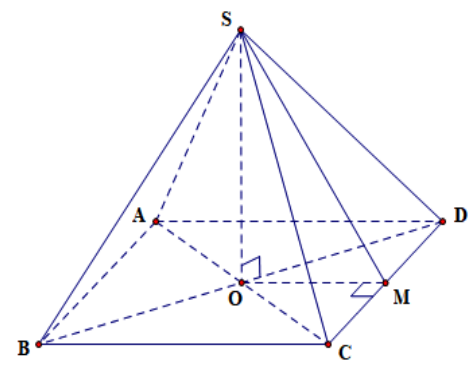

Solution

Let $O=A C \cap B D \Rightarrow S O \perp(A B C D), \quad M$ is a midpoint of $C D$

Thus, $S M^{2}=S D^{2}-D M^{2}=b^{2}-\frac{a^{2}}{4}$

In the other side, $\triangle S O M$ is a right triangular at $\mathrm{O}$, one has:

$S O=\sqrt{S M^{2}-O M^{2}}=\sqrt{b^{2}-\frac{a^{2}}{4}-\frac{a^{2}}{4}}=\frac{\sqrt{4 b^{2}-2 a^{2}}}{2}$

Hence we get, $V_{S . A B C D}=\frac{1}{3} \cdot S O \cdot S_{A B C D}=\frac{1}{3} \cdot \frac{\sqrt{4 b^{2}-2 a^{2}}}{2} \cdot a^{2}$ $=\frac{a^{2} \sqrt{4 b^{2}-2 a^{2}}}{6}$

In case of if $a=b \Rightarrow V=\frac{a^{3} \sqrt{2}}{6}$.

Formula 7: Given an equilateral quadrilateral pyramid $S . A B C D$ has its base edge with the length is $a$, the angle between the side surface and the base plane is $\alpha$ :

$V_{S . A B C D}=\frac{a^{3} \tan \alpha}{6}$

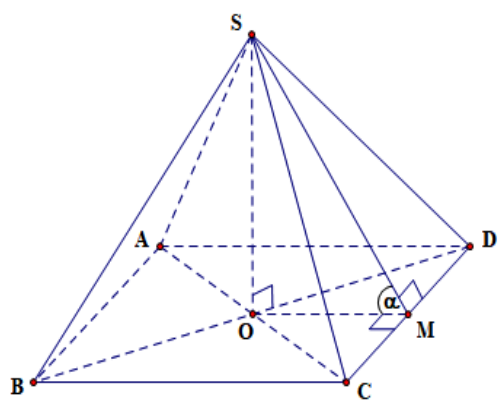

Solution

Let $O=A C \cap B D \Rightarrow S O \perp(A B C D)$, and $M \mathrm{M}$ is a midpoint of $C D$

Thus the angle of between $(S C D)$ and $(A B C D)$ is $\widehat{S M O}=\alpha$

Otherwise, one has $\triangle S O M$ is a right angle at $O$, thus $S O=O M \cdot \tan \alpha=\frac{a}{2} \cdot \tan \alpha$

Thereafter, we have: $V_{S \cdot A B C D}=\frac{1}{3} \cdot S O \cdot S_{A B C D}=\frac{1}{3} \cdot \frac{a}{2} \cdot \tan \alpha \cdot a^{2}$ $=\frac{a^{3} \tan \alpha}{6}$. 
Formula 8: Given an equilateral quadrilateral pyramid $S . A B C D$ has its base edge with the length is $a$, the angle between the side edge and the base plane is:

$$
V_{S . A B C D}=\frac{\sqrt{2} a^{3} \tan \beta}{6}
$$

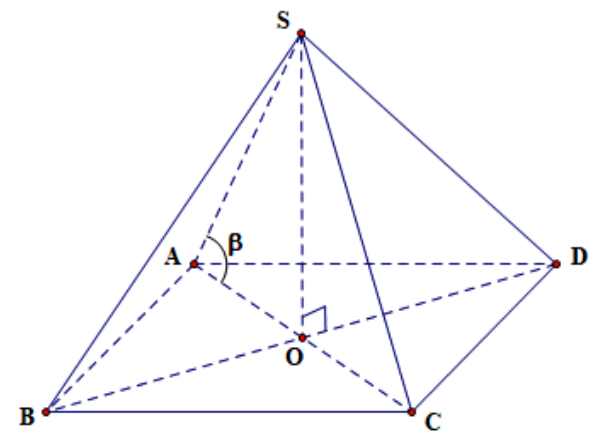

\section{Solution}

Let $O=A C \cap B D \Rightarrow S O \perp(A B C D)$

Thereafter, we get $A O=\frac{A C}{2}=\frac{a \sqrt{2}}{2}$

One has $\triangle S O A$ is a right triangular at $O$, thus $S O=A O \cdot \tan \beta=\frac{a \sqrt{2} \cdot \tan \beta}{2}$

Thereafter we obtain, $\quad V_{S . A B C D}=\frac{1}{3} \cdot S O \cdot S_{A B C D}$ $=\frac{1}{3} \cdot \frac{a \sqrt{2} \cdot \tan \beta}{2} \cdot a^{2}=\frac{\sqrt{2} a^{3} \tan \beta}{6}$.

Formula 9: Given an equilateral quadrilateral pyramid $S . A B C D$ has its base edge with the length is a, $\widehat{S A B}=\gamma$ with $\gamma \in\left(\frac{\pi}{4} ; \frac{\pi}{2}\right)$ :

$V_{S . A B C D}=\frac{a^{3} \sqrt{\tan ^{2} \gamma-1}}{6}$

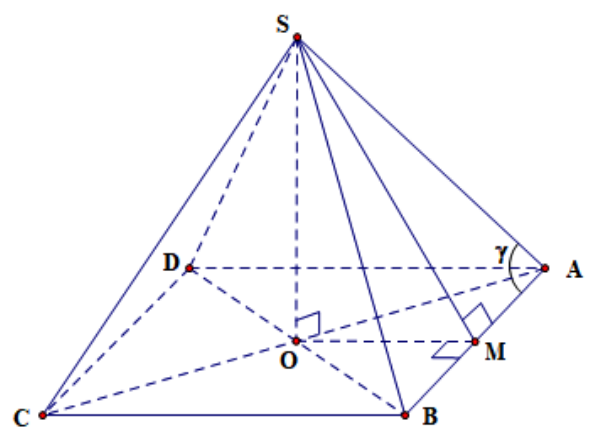

\section{Solution}

Let $O=A C \cap B D \Rightarrow S O \perp(A B C D)$ and $M$ is a midpoint of $A B$

$\triangle S M A$ is a right triangular at $M$, one has:

$S M=A M \cdot \tan \gamma=\frac{a \cdot \tan \gamma}{2}$

$\triangle S O M$ is a right triangular at $O$, thereafter we get:

$$
\begin{aligned}
& S O=\sqrt{S M^{2}-O M^{2}}=\sqrt{\frac{a^{2} \cdot \tan ^{2} \gamma}{4}-\frac{a^{2}}{4}}=\frac{a \sqrt{\tan ^{2} \gamma-1}}{2} \\
& \text { Thus, } V_{S . A B C D}=\frac{1}{3} \cdot S O \cdot S_{A B C D}=\frac{1}{3} \cdot \frac{a \sqrt{\tan ^{2} \gamma-1}}{2} \cdot a^{2} \\
& =\frac{a^{3} \sqrt{\tan ^{2} \gamma-1}}{6} .
\end{aligned}
$$

Formula 10: Given an equilateral quadrilateral pyramid $S . A B C D$ has its side edge with the length is a, the angle between the side surface and the base plane is $\alpha$ with $\alpha \in\left(0 ; \frac{\pi}{2}\right)$. Then one has:

$V_{S . A B C D}=\frac{4 b^{3} \tan \alpha}{3 \sqrt{\left(2+\tan ^{2} \alpha\right)^{3}}}$

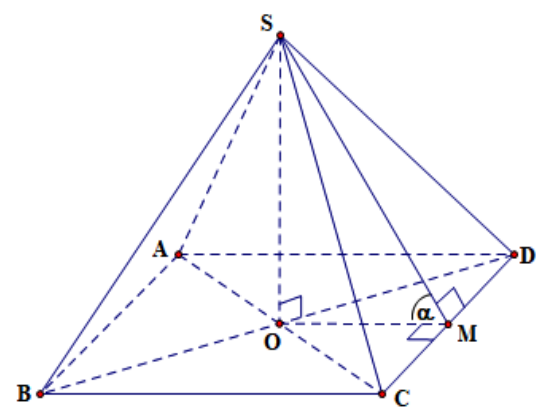

$\underline{\text { Solution }}$

Let $O=A C \cap B D \Rightarrow S O \perp(A B C D)$ and

$M$ is a midpoint of $C D$

Thus we have the angle of $(S C D)$ and $(A B C D)$ is $\widehat{S M O}=\alpha$

Let $x$ is the length of the base edge

It can be seen that $\triangle S M C$ is a right triangular at $M$, one has:

$S M=\sqrt{S C^{2}-C M^{2}}=\sqrt{b^{2}-\frac{x^{2}}{4}}$ 
In addition, one has $\triangle S O M$ is a right triangular at $O$ thus:

$O M=S M \cdot \cos \alpha \Rightarrow \frac{x}{2}=\sqrt{b^{2}-\frac{x^{2}}{4}} \cdot \cos \alpha \Rightarrow \frac{x^{2}}{4}=\left(b^{2}-\frac{x^{2}}{4}\right) \cdot \cos ^{2} \alpha$

$\Rightarrow x^{2}=\frac{4 b^{2} \cdot \cos \alpha}{1+\cos ^{2} \alpha}=\frac{4 b^{2} \cdot \frac{1}{1+\tan ^{2} \alpha}}{1+\frac{1}{1+\tan ^{2} \alpha}}=\frac{4 b^{2}}{2+\tan ^{2} \alpha}$

$\Rightarrow x=\frac{2 b}{\sqrt{2+\tan ^{2} \alpha}} \Rightarrow S_{A B C D}=\frac{4 b^{2}}{2+\tan ^{2} \alpha}$

Thereafter we get: $S O=O M \cdot \tan \alpha=\frac{x}{2} \cdot \tan \alpha$ $=\frac{b \cdot \tan \alpha}{\sqrt{2+\tan ^{2} \alpha}}$

Hence, we obtain $V_{S . A B C D}=\frac{1}{3} \cdot S O \cdot S_{A B C D}=\frac{1}{3} \cdot \frac{b \cdot \tan \alpha}{\sqrt{2+\tan ^{2} \alpha}}$ $\frac{4 b^{2}}{2+\tan ^{2} \alpha}=\frac{4 a^{3} \cdot \tan \alpha}{3 \sqrt{\left(2+\tan ^{2} \alpha\right)^{3}}}$.

\subsubsection{Normal Pyramids}

Formula 11: Given a pyramid $S . A B C$ has $S A$ perpendicular with $(A B C)$, and $(S A B)$ perpendicular with $(S B C), \widehat{B S C}=\alpha, \widehat{A S B}=\beta$ :

$V_{S . A B C}=\frac{S B^{3} \cdot \sin 2 \beta \cdot \tan \alpha}{12}$

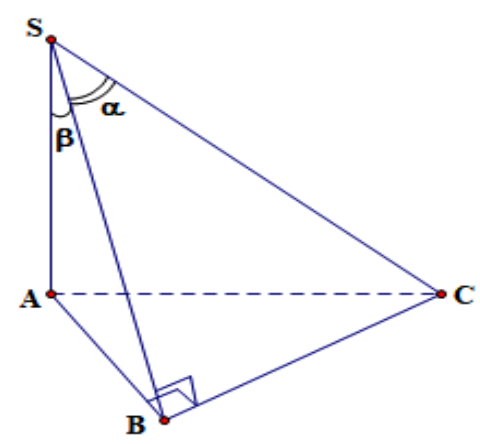

Solution

$\triangle S A B$ is a right triangular at $A$, one has:

$A B=S B \cdot \sin \beta, S A=S B \cdot \cos \beta$

It can be seen that, $\triangle S B C$ is a right triangular at $B$

In addition, we have: $B C=S B \cdot \tan \alpha$

$$
\begin{aligned}
\Rightarrow V_{S \cdot A B C}= & \frac{1}{3} \cdot S A \cdot S_{\triangle A B C}=\frac{1}{3} S A \cdot \frac{1}{2} \cdot A B \cdot B C \\
& =\frac{1}{6} \cdot S B \cdot \cos \beta \cdot S B \cdot \sin \beta \cdot S B \cdot \tan \alpha=\frac{S B^{3} \cdot \sin 2 \beta \cdot \tan \alpha}{12}
\end{aligned}
$$

\subsection{Tetrahedral Volume}

\subsubsection{Regular Tetrahedron}

Formula 12: Given a regular tetrahedron S.ABC with the length of its edge is a:

$V_{S . A B C}=\frac{a^{3} \sqrt{2}}{12}$

\subsubsection{Nearly Regular Tetrahedron}

Formula 13: Give a nearly regular tetrahedron:

$S A=B C=a$

$\left\{\begin{array}{l}S B=A C=b \\ S C=A B=c\end{array}\right.$

$V_{S . A B C}=\frac{\sqrt{2}}{12} \sqrt{\left(a^{2}+b^{2}-c^{2}\right)\left(b^{2}+c^{2}-a^{2}\right)\left(a^{2}+c^{2}-b^{2}\right)}$

\subsubsection{Square Tetrahedron}

Formula 14: Given a tetrahedron $S A B C$ has $(S A B)$, $(S B C),(S A C)$ perpendicular to each other in pairs, the triangle area of $S A B, S B C, S A C$ are $S_{1}, S_{2}, S_{3}$, respectively:

$V_{S . A B C}=\frac{\sqrt{2 S_{1} S_{2} S_{3}}}{3}$

Formula 15: Given a tetrahedron $S A B C$ has $S A, S B, S C$ perpendicular to each other in pairs and the length of them are $a, b, c$, respectively:

$V_{S . A B C}=\frac{a b c}{6}$

\subsubsection{Normal Tetrahedron}

Formula 16: Given the length of 3 edges and 3 degrees at the top:

$\left\{\begin{array}{l}S A=a, S B=b, S C=c \\ \widehat{A S B}=\alpha, \widehat{B S C}=\beta, \widehat{C S A}=\gamma\end{array}\right.$

$V_{S . A B C}=\frac{a b c}{6} \sqrt{1-\cos ^{2} \alpha-\cos ^{2} \beta-\cos ^{2} \gamma+2 \cos \alpha \cos \beta \cos \gamma}$

Formula 17: Given the length of the opposite sides, the distance and the angle of that two sides: 


$$
\begin{aligned}
& \left\{\begin{array}{l}
S A=a, B C=b \\
d(S A, B C)=d ;(S A, B C)=\alpha
\end{array}\right. \\
& V_{S . A B C}=\frac{1}{6} a b d \sin \alpha
\end{aligned}
$$

Formula 18: Given the length of an edge, area and angle between two adjacent surfaces:

$$
\begin{gathered}
\left\{\begin{array}{l}
S_{\triangle S A B}=S_{1}, S_{\triangle S A C}=S_{2}, S A=a \\
((S A B),(S A C))=\alpha
\end{array}\right. \\
V_{S . A B C}=\frac{2 S_{1} S_{2} \sin \alpha}{3 a}
\end{gathered}
$$

Formula 19: Given the length of 3 edges, 2 vertices and 1 dihedral angle:

$$
\begin{aligned}
& \left\{\begin{array}{l}
S A=a, S B=b, S C=c \\
((S A B),(S A C))=\alpha \\
\widehat{A S B}=\beta, \widehat{A S C}=\gamma
\end{array}\right. \\
& V_{S . A B C}=\frac{a b c}{6} \sin \alpha \sin \beta \sin \gamma .
\end{aligned}
$$

\subsection{Volume of Other Polyhedral}

Formula 20: The octahedron has vertices that centers of the sides of the cube with the length of its edge is a:

$$
V=\frac{a^{3}}{6}
$$

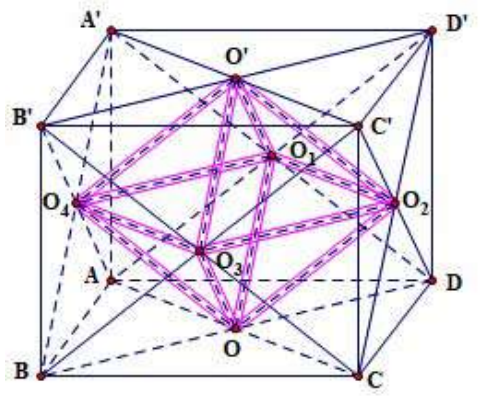

Solution

The length of the hight line of $\mathrm{O}^{\prime} \mathrm{O}_{1} \mathrm{O}_{2} \mathrm{O}_{3} \mathrm{O}_{4}$ is $h=\frac{O O^{\prime}}{2}=\frac{a}{2}$

Thereafter, we get: $\quad O_{2} O_{3}=\frac{B D}{2}=\frac{a \sqrt{2}}{2}$ $\Rightarrow S_{O_{1} O_{2} O_{3} O_{4}}=\left(\frac{a \sqrt{2}}{2}\right)^{2}=\frac{a^{2}}{2}$
Thus, we obtain $V_{O^{\prime} O_{1} O_{2} O_{3} O_{4} O}=2 V_{O^{\prime} O_{1} O_{2} O_{3} O_{4}}=2 \cdot \frac{1}{3} \cdot h \cdot S_{O_{1} O_{2} O_{3} O_{4}}$ $=2 \cdot \frac{1}{3} \cdot \frac{a}{2} \cdot \frac{a^{2}}{2}=\frac{a^{3}}{6}$.

Formula 21: Given a regular octahedral with the length of its edge is a. Connect the center of side surfaces to the cube:

$V=\left(\frac{a \sqrt{2}}{3}\right)^{3}=\frac{2 a^{3} \sqrt{2}}{27}$

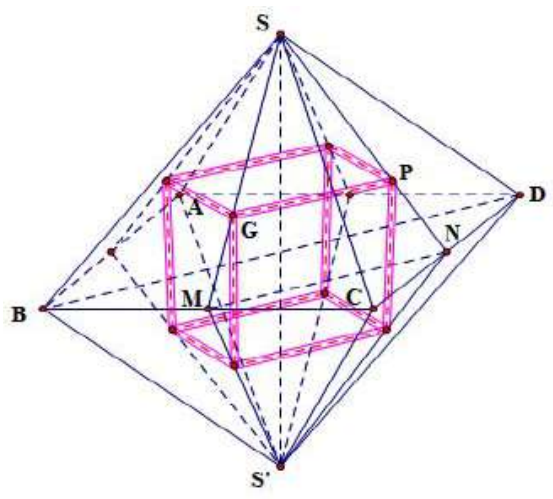

Solution:

It can be seen that $G P=\frac{2}{3} M N=\frac{1}{3} B D=\frac{a \sqrt{2}}{3}$

So the volume of the cube $V=\left(\frac{a \sqrt{2}}{3}\right)^{3}=\frac{2 \sqrt{2} a^{3}}{27}$.

Formula 22: The regular dodecahedron with the length of its edge is a. Then one has:

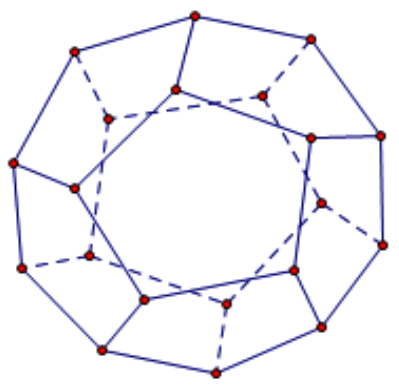

$V=\frac{a^{3}(15+7 \sqrt{5})}{4}$

In order to evaluate efficiently of the proposed formulas, we next turn on investigate to empirical analysis at Can Tho City in the next section. 


\section{EMPIRICAL ANALYSIS AT CAN THO CITY}

\subsection{Objectives and Tasks Experiment}

\subsubsection{Objectives Experiment}

In this paper, we evaluate 40 objective multiple choice questions with 4 options on the subject volume of polyhedron by IATA software. We aim to compare traditional work with the work has the support of quick calculation formula.

\subsubsection{Tasks Experiment}

We first build test questions and a summary table of recipes for experiment, and then we obtain the results of student work to data analysis. Finally, we evaluate experimental results.

\subsection{Content and Empirical Process}

\subsubsection{Experimental Objects}

Test questions are experimented on subjects who have learned through chapter 2 , geometry 12 . We have surveyed 175 students at Nguyen Viet Hong High School, Chau Van Liem High School, Phan Van Tri High School, Ly Tu Trong High School for the Gifted, High School Pedagogical Practice, Phan Ngoc Hien High School, An Khanh High School, Nga Sau High School, Bui Huu Nghia High School, Luu Huu Phuoc High School.

\subsubsection{Structure of Experimental Topics}

\begin{tabular}{|c|c|}
\hline Equilateral triangular pyramid & $\begin{array}{l}\text { Formula 1: Question 1, } 2 . \\
\text { Formula 2: Question 3, } 4 . \\
\text { Formula 3: Question 5, } 6 . \\
\text { Formula 4: Question 7, } 8 . \\
\text { Formula 5: Question 9, } 10 .\end{array}$ \\
\hline Quadrilateral pyramids & $\begin{array}{l}\text { Formula 6: Question 11, } 12 . \\
\text { Formula 7: Question 13, } 14 . \\
\text { Formula 8: Question 15, } 16 . \\
\text { Formula 9: Question 17. } \\
\text { Formula 10: Question 18, } 19 .\end{array}$ \\
\hline Normal pyramids & Formula 11: Question 20, 21. \\
\hline Regular tetrahedron & Formula 12: Question 22, 23. \\
\hline Nearly regular tetrahedron & Formula 13: Question 24, 25. \\
\hline Square tetrahedron & $\begin{array}{l}\text { Formula 14: Question 26, } 27 . \\
\text { Formula 15: Question 28, } 29 .\end{array}$ \\
\hline Normal tetrahedron & $\begin{array}{l}\text { Formula 16: Question 30, } 31 . \\
\text { Formula 17: Question 32, } 33 . \\
\text { Formula 18: Question 34, } 35 . \\
\text { Formula 19: Question 36, } 37 .\end{array}$ \\
\hline Other polyhedrons & $\begin{array}{l}\text { Formula 20: Question } 38 . \\
\text { Formula 21: Question } 39 . \\
\text { Formula 22: Question } 40 .\end{array}$ \\
\hline
\end{tabular}

\subsubsection{Experimental Subject}

Question 1: Given an equilateral triangular pyramid $S . A B C$ has its base is an equilateral triangle $A B C$ with the length of its edge is $a$, the length of the side edge is $\sqrt{3} a$. Volume of pyramid $S . A B C$ will equal to:
A. $\frac{a^{3}}{4}$.
B. $\frac{a^{3} \sqrt{2}}{6}$.
C. $\frac{a^{3} \sqrt{2}}{4}$.
D. $a^{3} 2 \sqrt{2}$.

Question 2: Given an equilateral triangular pyramid $S . A B C$ has its base is an equilateral triangle $A B C$ with the length of its edge is $a$, the side edge with the length is $2 a$. Volume of pyramid $S . A B C$ is:
A. $\frac{a^{3}}{3}$
B. $a^{3} \sqrt{11}$.
C. $\frac{a^{3} \sqrt{2}}{3}$.
D. $\frac{a^{3} \sqrt{11}}{12}$.

Question 3: Given an equilateral triangular pyramid $S . A B C$ has the length of its base edge is $a$ and the side surface creates a base plane at an angle of $60^{\circ}$. Volume of pyramid $S . A B C$ is given by:
A. $\frac{a^{3} \sqrt{3}}{12}$.
B. $\frac{a^{3}}{24}$.
C. $\frac{a^{3} \sqrt{3}}{24}$.
D. $\frac{a^{3}}{48}$

Question 4: Given an equilateral triangular pyramid $S . A B C$ has its base edge with the length is $a \sqrt{2}$ and the side surface creates a base plane at an angle of $30^{\circ}$. Volume of pyramid $S . A B C$ is provided by:
A. $\frac{a^{3} \sqrt{6}}{36}$.
B. $\frac{a^{3} \sqrt{3}}{36}$.
C. $\frac{a^{3} \sqrt{6}}{18}$.
D. $\frac{a^{3} \sqrt{2}}{12}$

Question 5: Given an equilateral triangular pyramid $S . A B C$ has the length of its base edge is $a \sqrt{2}$ and the side edge creates a base plane at an angle of $60^{\circ}$. Volume of pyramid is illustrated by:
A. $\frac{a^{3} 3 \sqrt{2}}{16}$.
B. $\frac{a^{3} 3 \sqrt{2}}{4}$.
C. $\frac{a^{3} 3 \sqrt{2}}{8}$.
D. $\frac{a^{3} 3 \sqrt{6}}{16}$.

Question 6: Given an equilateral triangular pyramid $S . A B C$ has its base edge with the length is $a$ and the side edge creates a base plane at an angle of $30^{\circ}$. Volume of pyramid will equal to:
A. $\frac{3 a^{3}}{16}$.
B. $\frac{3 \sqrt{3} a^{3}}{8}$.
C. $\frac{3 \sqrt{3} a^{3}}{32}$.
D. $\frac{3 a^{3}}{32}$.

Question 7: Given an equilateral triangular pyramid $S . A B C$ has the length of its base edge is $2 a$ and the side edge creates a base plane at an angle of $30^{\circ}$. Volume of pyramid $S . A B C$ is: 

A. $\frac{a^{3} \sqrt{3}}{36}$.
B. $\frac{a^{3} \sqrt{3}}{18}$.
C. $\frac{a^{3} \sqrt{3}}{9}$.
D. $\frac{a^{3} 2 \sqrt{3}}{9}$.
A. $\frac{a^{3} \sqrt{3}}{64}$.
B. $\frac{a^{3} \sqrt{6}}{16}$.
C. $\frac{a^{3} \sqrt{3}}{16}$.
D. $\frac{a^{3} \sqrt{3}}{12}$.

Question 8: Given an equilateral triangular pyramid $S . A B C$ has the length of its base edge is $a$ and the side edge creates a base plane at an angle of $60^{\circ}$. Volume of pyramid $S . A B C$ is given by:
A. $\frac{a^{3} \sqrt{3}}{24}$.
B. $\frac{a^{3} \sqrt{3}}{12}$.
C. $\frac{3 a^{3}}{32}$.
D. $\sqrt{3} a^{3}$.

Question 9: Given an equilateral triangular pyramid $S . A B C$ has its base edge with the length is $a$. Let $(P)$ is the plane passing through $A$ parallel to $B C$ and perpendicular to $(S B C)$, the angle between the plane $(P)$ and the base plane is $30^{\circ}$. Volume of pyramid $S . A B C$ is provided by:
A. $\frac{a^{3} \sqrt{3}}{72}$.
B. $\frac{a^{3} \sqrt{3}}{48}$.
C. $a^{3} \sqrt{3}$.
D. $\frac{a^{3} \sqrt{3}}{24}$.

Question 10: Given an equilateral triangular pyramid $S . A B C$ has the length of its base edge is $2 a$. Let $(P)$ is the plane passing through $A$ parallel to $B C$ and perpendicular to $(S B C)$, the angle between the plane $(P)$ and the base plane is $60^{\circ}$. Volume of pyramid $S . A B C$ is illustrated by:
A. $\frac{a^{3} \sqrt{3}}{36}$.
B. $\frac{a^{3}}{6}$.
C. $\frac{a^{3} \sqrt{3}}{9}$.
D. $\frac{a^{3} \sqrt{3}}{3}$.

Question 11: Given equilateral quadrilateral pyramid $S . A B C D$ has base plane is a square $A B C D$ with the length of its edge is $a$ and the length of the side edge is $2 a$. Volume of pyramid $S . A B C D$ will equal to:
A. $\frac{a^{3} \sqrt{14}}{6}$.
B. $\frac{a^{3} \sqrt{2}}{2}$.
C. $\frac{a^{3} \sqrt{2}}{6}$.
D. $\frac{a^{3} \sqrt{6}}{6}$.

Question 12: Given equilateral quadrilateral pyramid $S . A B C D$ have all edges equal to $2 a$. Volume of pyramid $S . A B C D$ is:
A. $\frac{a^{3} \sqrt{2}}{6}$.
B. $\frac{a^{3} 4 \sqrt{2}}{3}$.
C. $\frac{a^{3} \sqrt{2}}{3}$.
D. $\frac{a^{3} 2 \sqrt{2}}{3}$.

Question 13: Given equilateral quadrilateral pyramid $S . A B C D$ has its base edge with the length is $\frac{a \sqrt{3}}{2}$, the side surface creates a base plane at an angle of $45^{\circ}$. Volume of pyramid is given by:

Question 14: Given equilateral quadrilateral pyramid $S . A B C D$ has the length of its base edge is $2 a$, the side surface creates a base plane at an angle of $60^{\circ}$. Volume of pyramid is provided by:
A. $\frac{a^{3} \sqrt{3}}{3}$.
B. $\frac{a^{3} 4 \sqrt{6}}{3}$.
C. $\frac{a^{3} \sqrt{3}}{6}$.
D. $\frac{a^{3} 4 \sqrt{3}}{3}$.

Question 15: Given equilateral quadrilateral pyramid $S . A B C D$ has its base edge with the length is $a \sqrt{3}$, the side edge creates a base plane at an angle of $30^{\circ}$. Volume of pyramid $S . A B C D$ is illustrated by:
A. $\frac{a^{3} \sqrt{6}}{18}$.
B. $\frac{a^{3}}{4}$.
C. $\frac{a^{3}}{2}$.
D. $\frac{a^{3} \sqrt{2}}{2}$.

Question 16: Given equilateral quadrilateral pyramid $S . A B C D$ has the length of its base edge is $2 a$, the side edge creates a base plane at an angle of $45^{\circ}$. Volume of pyramid $S . A B C D$ will equal to:
A. $\frac{a^{3} \sqrt{2}}{3}$.
B. $\frac{a^{3} 4 \sqrt{2}}{3}$.
C. $\frac{a^{3} \sqrt{2}}{6}$.
D. $\frac{4 a^{3}}{3}$.

Question 17: Given equilateral quadrilateral pyramid $S . A B C D$ has its base edge with the length is $a, \widehat{S A B}=60^{\circ}$. Volume of pyramid $S . A B C D$ is:
A. $\frac{a^{3} \sqrt{-1+\sqrt{3}}}{6}$.
B. $a^{3} \sqrt{2}$.
C. $\frac{a^{3} \sqrt{2}}{6}$.
D. $\frac{a^{3}}{3}$.

Question 18: Given equilateral quadrilateral pyramid $S . A B C D$ has the length of its side edge is $2 a$, the side surface creates a base plane at an angle of $45^{\circ}$. Volume of pyramid $S . A B C D$ is given by:
A. $\frac{a^{3} 32 \sqrt{3}}{27}$.
B. $\frac{a^{3} 8 \sqrt{3}}{27}$.
C. $\frac{a^{3} 4 \sqrt{3}}{27}$.
D. $\frac{a^{3} 32 \sqrt{3}}{9}$.

Question 19: Given equilateral quadrilateral pyramid $S . A B C D$ has its side edge with the length is $a$, the side surface creates a base plane at an angle of $60^{\circ}$. Volume of pyramid $S . A B C D$ is provided by:
A. $\frac{a^{3} \sqrt{15}}{75}$.
B. $\frac{a^{3} 4 \sqrt{15}}{75}$.
C. $\frac{a^{3} 4 \sqrt{15}}{15}$.
D. $\frac{a^{3} 4 \sqrt{15}}{25}$.

Question 20: Given a pyramid $S . A B C$ has $S A$ perpendicular to $(A B C),(S A B)$ perpendicular to $(S B C), \quad S B=a \sqrt{3}, \quad \widehat{B S C}=45^{\circ}, \widehat{A S B}=30^{\circ}$. Volume of pyramid $S . A B C D$ is illustrated by: 

A. $\frac{3 a^{3}}{8}$.
B. $\frac{a^{3} \sqrt{3}}{8}$.
C. $\frac{a^{3}}{4}$.
D. $\frac{9 a^{3}}{2}$.
A. $\frac{a^{3} 2 \sqrt{3}}{3}$.
B. $a^{3} 2 \sqrt{3}$.
C. $\frac{a^{3} \sqrt{6}}{3}$.
D. $a^{3}$.

Question 21: Given a pyramid $S . A B C$ has $S A$ perpendicular to $(A B C)$, two planes $(S A B)$ and $(S B C)$ are perpendicular to together, $S B=a$, $\widehat{B S C}=30^{\circ}, \widehat{A S B}=60^{\circ}$. Volume of pyramid $S . A B C$ will equal to:
A. $\frac{a^{3} \sqrt{3}}{24}$.
B. $\frac{a^{3}}{8}$.
C. $\frac{a^{3}}{24}$.
D. $\frac{a^{3}}{2}$.

Question 22: Given regular tetrahedron $S . A B C$ has all edges equal to $a \sqrt{2}$. Volume of pyramid $S . A B C$ is:
A. $\frac{a^{3} \sqrt{6}}{4}$.
B. $\frac{a^{3} \sqrt{2}}{6}$.
C. $\frac{a^{3} \sqrt{2}}{12}$.
D. $\frac{a^{3}}{3}$.

Question 23: Given regular tetrahedron $S . A B C$ has all edges equal to $2 a$. Volume of pyramid $S . A B C$ is given by:
A. $\frac{a^{3} \sqrt{2}}{12}$.
B. $\frac{a^{3} 2 \sqrt{2}}{3}$.
C. $\frac{a^{3} \sqrt{2}}{6}$.
D. $\frac{a^{3} 3 \sqrt{2}}{2}$.

Question 24: Given a tetrahedron S.ABC has $S A=B C=4 a, S B=A C=5 a, \quad S C=A B=6 a$. Volume of pyramid $S . A B C$ is provided by:
A. $\frac{a^{3} 15 \sqrt{6}}{4}$.
B. $\frac{a^{3} 77 \sqrt{154}}{12}$.
C. $a^{3} 45 \sqrt{3}$.
D. $\frac{a^{3} \sqrt{154}}{12}$.

Question 25: Given a tetrahedron $S . A B C$ has $S A=B C=6 \mathrm{~cm}, S B=A C=7 \mathrm{~cm}, S C=A B=8 \mathrm{~cm}$. Volume of pyramid $S . A B C$ is illustrated by:
A. $21 \sqrt{187} \mathrm{~cm}^{3}$.
B. $\frac{\sqrt{298}}{12} \mathrm{~cm}^{3}$.
C. $\frac{7 \sqrt{374}}{4} \mathrm{~cm}^{3}$.
D. $\frac{27489 \sqrt{2}}{4} \mathrm{~cm}^{3}$.

Question 26: Given a tetrahedron S.ABC has planes $(S A B),(S B C),(S A C)$ perpendicular to each other in pairs, area of triangles $S A B, S B C, S A C$ are $15 \mathrm{~cm}^{2}, 20 \mathrm{~cm}^{2}, 18 \mathrm{~cm}^{2}$, respectively. Volume of pyramid $S . A B C$ will equal to:
A. $60 \sqrt{3} \mathrm{~cm}^{3}$.
B. $10 \sqrt{6} \mathrm{~cm}^{3}$.
C.
$900 \mathrm{~cm}^{3}$.
D. $20 \sqrt{3} \mathrm{~cm}^{3}$.

Question 27: Given a tetrahedron S.ABC has planes $(S A B),(S B C),(S A C)$ perpendicular to each other in pairs, area of triangles $S A B, S B C, S A C$ are $a^{2}, 2 a^{2}, 3 a^{2}$, respectively. Volume of pyramid $S . A B C$ is:

Question 28: Given a tetrahedron $S . A B C$ has $S A, S B, S C$ perpendicular to each other in pairs and its length are $5 \mathrm{~cm}, 10 \mathrm{~cm}, 2 \mathrm{dm}$, respectively. Volume of pyramid $S . A B C$ is given by:
A. $\frac{50}{3} \mathrm{~cm}^{3}$.
B. $\frac{500}{3} \mathrm{~cm}^{3}$.
C. $\frac{20 \sqrt{5}}{3} \mathrm{~cm}^{3}$.
D. $\frac{35}{6} \mathrm{~cm}^{3}$.

Question 29: Given a tetrahedron $S . A B C$ has $S A, S B, S C$ perpendicular to each other in pairs and its length are $3 a, 2 a, a$, respectively. Volume of pyramid $S . A B C$ is provided by:
A. $6 a^{3}$.
B. $\frac{5}{6} a^{3}$.
C. $a^{3}$.
D. $\frac{2 \sqrt{3}}{3} a^{3}$.

Question 30: Given a tetrahedron $S . A B C$ has $S A=S B=S C=a, \widehat{A S B}=\widehat{B S C}=60^{\circ}, \widehat{C S A}=90^{\circ}$. Volume of pyramid $S . A B C$ is illustrated by:
A. 0 .
B. $\frac{a^{3} \sqrt{2}}{12}$.
C. $\frac{a^{3} \sqrt{6}}{12}$
D. $\frac{a^{3}}{12}$.

Question 31: Given a tetrahedron $S . A B C$ has $S A=S B=a, S C=2 a, \widehat{A S B}=60^{\circ}, \widehat{B S C}=90^{\circ}, \widehat{C S A}=120^{\circ}$ Volume of pyramid $S . A B C$ will equal to:
A. $\frac{a^{3} \sqrt{6}}{6}$.
B. 0 .
C. $\frac{a^{3} \sqrt{2}}{6}$.
D. $\frac{a^{3}}{6}$.

Question 32: Given a tetrahedron $S . A B C$ has $S A=a, B C=a \sqrt{3}$, the distance between two straight lines $S A$ and $B C$ has the length of $8 a$ and the angle between them equal to $60^{\circ}$. Volume of pyramid $S . A B C$ is:
A. $2 a^{3}$.
B. $\frac{a^{3} 2 \sqrt{3}}{3}$.
C. $12 a^{3}$.
D. $\frac{a^{3}}{4}$.

Question 33: Given a tetrahedron $S . A B C$ has $S A=B C=3 a$, two straight lines $S A$ and $B C$ perpendicular to each other and the distance between them equal to $2 a$. Volume of pyramid $S . A B C$ is given by:
A. $a^{3}$.
B. $\frac{3}{2} a^{3}$.
C. $18 a^{3}$.
D. $3 a^{3}$.

Question 34: Given a tetrahedron $S . A B C$ has $S A=2 a$, area of triangles of $S A B$ and $S A C$ are 
$2 a^{2}, 5 a^{2}$, respectively and the angle between two planes $(S A B)$ and $(S A C)$ equal to $30^{\circ}$. Volume of pyramid $S . A B C$ is provided by:
A. $5 a^{3}$.
B. $\frac{5}{3} a^{3}$.
C. $\frac{5}{6} a^{3}$.
D. $\frac{10}{3} a^{3}$.

Question 35: Given a tetrahedron $S . A B C$ has $S A=a \sqrt{2}$, area of triangles of $S A B$ and $S A C$ are $\frac{\sqrt{6}}{2} a^{2}, \frac{\sqrt{3}}{2} a^{2}$, respectively and the angle between two planes $(S A B)$ and $(S A C)$ equal to $60^{\circ}$. Volume of pyramid $S . A B C$ is illustrated by:
A. $\frac{a^{3} \sqrt{3}}{4}$.
B. $\frac{a^{3} 3 \sqrt{3}}{4}$.
C. $\frac{a^{3} \sqrt{6}}{4}$.
D. $\frac{a^{3} \sqrt{3}}{8}$.

Question 36: Given a tetrahedron $S . A B C$ has $S A=2 a, S B=3 a, S C=5 a$, and the angle between two planes $(S A B)$ and $(S A C)$ is $30^{\circ}$ and $\widehat{A S B}=15^{\circ}, \widehat{A S C}=45^{\circ}$. Volume of pyramid $S . A B C$ will equal to:
A. $\frac{-15+15 \sqrt{3}}{4} a^{3}$.
B. $\frac{-5+5 \sqrt{3}}{8} a^{3}$.
C. $\frac{-5+5 \sqrt{3}}{24} a^{3}$.
D. $\frac{15+5 \sqrt{3}}{8} a^{3}$.

Question 37: Given a tetrahedron $S . A B C$ has $S A=a, S B=a \sqrt{2}, S C=a \sqrt{3}$, and the angle between two planes $(S A B)$ and $(S A C)$ is $60^{\circ}$ and $\widehat{A S B}=30^{\circ}, \widehat{A S C}=45^{\circ}$. Volume of pyramid $S . A B C$ is:
A. $\frac{3 a^{3}}{4}$.
B. $\frac{a^{3}}{8}$.
C. $\frac{a^{3} \sqrt{6}}{8}$.
D. $\frac{a^{3} \sqrt{6}}{48}$.

Question 38: Given a regular octahedron has the vertex is the center of the sides of the cube with its length is $2 a$. Volume of the regular dodecahedron is given by:
A. $\frac{4 a^{3}}{3}$.
B. $\frac{a^{3}}{3}$.
C. $\frac{2 a^{3}}{3}$.
D. $\frac{a^{3}}{6}$.

Question 39: Given a regular octahedron with the length of its edge $a$. Connecting the center of its sides will be a cube with volume $V$. The score of $\frac{a^{3}}{V}$ is provided by :
A. 6 .
B. $\frac{1}{6}$.
C. $\frac{2 \sqrt{2}}{27}$.
D. $\frac{27 \sqrt{2}}{4}$.

Question 40: The regular dodecahedron with the length of its edge is $2 a$, its volume is illustrated by:
A.
B.
C.
D.

$$
\frac{15+7 \sqrt{5}}{2} a^{3} . \quad(15+7 \sqrt{5}) a^{3} . \quad(30+14 \sqrt{5}) a^{3} . \quad \frac{15+7 \sqrt{5}}{4} a^{3} .
$$

The answers of the above 40 questions are provided in the following table:

\begin{tabular}{|c|c|c|c|c|c|c|c|c|c|c|c|c|c|c|c|c|c|c|c|}
\hline 1 & 2 & 3 & 4 & 5 & 6 & 7 & 8 & 9 & 10 & 11 & 12 & 13 & 14 & 15 & 16 & 17 & 18 & 19 & 20 \\
\hline $\mathrm{B}$ & $\mathrm{D}$ & $\mathrm{C}$ & $\mathrm{A}$ & $\mathrm{A}$ & $\mathrm{C}$ & $\mathrm{D}$ & $\mathrm{B}$ & $\mathrm{D}$ & $\mathrm{C}$ & $\mathrm{A}$ & $\mathrm{B}$ & $\mathrm{C}$ & $\mathrm{D}$ & $\mathrm{D}$ & $\mathrm{B}$ & $\mathrm{C}$ & $\mathrm{A}$ & $\mathrm{B}$ & $\mathrm{A}$ \\
\hline 21 & 22 & 23 & 24 & 25 & 26 & 27 & 28 & 29 & 30 & 31 & 32 & 33 & 34 & 35 & 36 & 37 & 38 & 39 & 40 \\
\hline $\mathrm{C}$ & $\mathrm{D}$ & $\mathrm{B}$ & $\mathrm{A}$ & $\mathrm{C}$ & $\mathrm{D}$ & $\mathrm{A}$ & $\mathrm{B}$ & $\mathrm{C}$ & $\mathrm{B}$ & $\mathrm{C}$ & $\mathrm{A}$ & $\mathrm{D}$ & $\mathrm{B}$ & $\mathrm{A}$ & $\mathrm{B}$ & $\mathrm{B}$ & $\mathrm{A}$ & $\mathrm{D}$ & $\mathrm{C}$ \\
\hline
\end{tabular}

After designing multiple choice questions, we perform an experimental process, we present this issue in the next sub-section.

\subsubsection{Experimental Process}

Experiments are designed in 2 approaches:

\section{Approach 1}

The main aim is to assess 40 multiple choice questions in the experiment using IATA software. With this objective we conduct to 120 students to solve multiple choice tests within 90 minutes.

\section{Approach 2}

The primary objective is to evaluate the effectiveness of formulas for quick calculation of volume in math problems. With this target we choose a control group (group A) and experimental group (group B) and jointly conduct a multiple choice test, with the require that group $B$ use the formula table, while group A does not use it.

Before conducting the experiments, we have studied the learning results of the groups. The results of the first semester study of the experimental group and the control group are listed in the following table. 
Table 1: Average Score of the First Semester of Mathematics of the Experimental Group and the Control Group

\begin{tabular}{|c|c|c|c|c|}
\hline \multirow{2}{*}{ Scores } & \multicolumn{2}{|c|}{ Experimental group (Group B) } & \multicolumn{2}{|c|}{ Control group (Group A) } \\
\cline { 2 - 5 } & Quantity & Proportion & Quantity & 37 \\
\hline \hline $8.0-10.0$ & 18 & $45 \%$ & 0 & 0 \\
\hline $6.5-7.9$ & 22 & $55 \%$ & 0 & 0 \\
\hline $5.0-6.4$ & 0 & 0 & 0 & 0 \\
\hline Under 5.0 & 0 & 0 & 37 & $100 \%$ \\
\hline Total & 40 & $100 \%$ & & 0 \\
\hline
\end{tabular}

The first step shows that the control group has a high mathematics score: $100 \%$ good. At that time, the experimental group was lower than the control group: $45 \%$ good and $55 \%$ rather good. In order to evaluate comprehensively a student's test, we need prior and posterior analysis. We first discuss the prior analysis in the next sub-section.

\subsection{Prior Analysis}

\section{Question 1}

The regular tetrahedral volume is

$V_{S . A B C}=\frac{a^{2} \sqrt{3 b^{2}-a^{2}}}{12}=\frac{a^{2} \sqrt{3 \cdot(\sqrt{3} a)^{2}-a^{2}}}{12}=\frac{a^{3} \sqrt{2}}{6}$

So choosing Answer B.

- $\quad$ Answer $\mathrm{A}$ is wrong because students forget to minus $a^{2}$ in the formula.

- $\quad$ Answer $C$ is wrong because students mistake $\frac{(\sqrt{3} a)^{2} \sqrt{3 a^{2}-a^{2}}}{12}$.

- $\quad$ Answer $D$ is wrong because students neglect to divide 12.

\section{$\underline{\text { Question } 2}$}

The regular tetrahedral volume is

$V_{S . A B C}=\frac{a^{2} \sqrt{3 b^{2}-a^{2}}}{12}=\frac{a^{2} \sqrt{3 \cdot(2 a)^{2}-a^{2}}}{12}=\frac{a^{3} \sqrt{11}}{12}$

Hence selecting Answer D.

- $\quad$ Answer $\mathrm{A}$ is wrong because students forget to replace $2 a^{2}$.

- $\quad$ Answer $B$ is wrong because students neglect to divide 12.
- Answer $\mathrm{C}$ is wrong because students mistake $\frac{(2 a)^{2} \sqrt{3 a^{2}-a}}{12}$.

\section{$\underline{\text { Question } 3}$}

$V_{S . A B C}=\frac{a^{3} \tan \alpha}{24}=\frac{a^{3} \cdot \tan 60^{\circ}}{24}=\frac{a^{3} \sqrt{3}}{24}$

Thus choosing Answer C.

- $\quad$ Answer $A$ is wrong because students used the wrong formula should only be divided by 12 .

- $\quad$ Answer B is wrong because students forget to multiply $\tan \alpha$.

- Answer $D$ is wrong because students mistake $\cos 60^{\circ}$.

\section{Question 4}

$V_{S . A B C}=\frac{a^{3} \tan \alpha}{24}=\frac{(a \sqrt{2})^{3} \cdot \tan 30^{\circ}}{24}=\frac{a^{3} \sqrt{6}}{36}$

Therefore, selecting Answer A.

- $\quad$ Answer B is wrong because of only replace $2 a^{3}$.

- $\quad$ Answer $C$ is wrong because students used the wrong formula should only be divided by 12 .

- $\quad$ Answer $D$ is wrong because students forget to multiply $\tan \alpha$.

$\underline{\text { Question } 5}$

$V_{S . A B C}=\frac{\sqrt{3} b^{3} \sin \beta \cos ^{2} \beta}{4}=\frac{\sqrt{3} \cdot(a \sqrt{2})^{3} \cdot \sin 60^{\circ} \cdot \cos ^{2} 60^{\circ}}{4}=\frac{a^{3} 3 \sqrt{2}}{16}$

So, choosing Answer A.

- $\quad$ Answer B is wrong because students neglect to divide 4 . 
- $\quad$ Answer $\mathrm{C}$ is wrong because students forget to square $\cos 60^{\circ}$.

- $\quad$ Answer $D$ is wrong because students use to square $\sin 60^{\circ}$ and students forget to square $\cos 60^{\circ}$.

\section{Question 6}

$V_{S . A B C}=\frac{\sqrt{3} b^{3} \sin \beta \cos ^{2} \beta}{4}=\frac{\sqrt{3} \cdot a^{3} \cdot \sin 30^{\circ} \cdot \cos ^{2} 30^{\circ}}{4}=\frac{a^{3} 3 \sqrt{3}}{32}$

Hence, selecting Answer C.

- $\quad$ Answer $A$ is wrong because students neglect to square $\cos 30^{\circ}$.

- $\quad$ Answer $B$ is wrong because students forget to divide 4 in the formula.

- $\quad$ Answer $D$ is wrong because of only replace edge length with $a$.

\section{Question 7}

$V_{S . A B C}=\frac{a^{3} \tan \beta}{12}=\frac{(2 a)^{3} \cdot \tan 30^{\circ}}{12}=\frac{a^{3} 2 \sqrt{3}}{9}$

Thus, choosing Answer D.

- $\quad$ Answer $A$ is wrong because of only replace edge length with $a$.

- $\quad$ Answer B is wrong because of only substituting $2 a^{3}$.

- $\quad$ Answer $\mathrm{C}$ is wrong because students mistake the formula, thus they divided to 24 in the formula.

\section{Question 8}

$$
V_{S . A B C}=\frac{a^{3} \tan \beta}{12}=\frac{a^{3} \cdot \tan 60^{\circ}}{12}=\frac{a^{3} \sqrt{3}}{12}
$$

Therefore selecting Answer B.

- $\quad$ Answer $\mathrm{A}$ is wrong because students mistake the formula, thus they divided to 24 in the formula.

- $\quad$ Answer $C$ is wrong because students mistake the formula: $\frac{\sqrt{3} \cdot a^{3} \cdot \sin 60^{\circ} \cdot \cos ^{2} 60^{\circ}}{4}$

- $\quad$ Answer $D$ is wrong because students forget to divide 12 in the formula. $\underline{\text { Question } 9}$

$V_{S . A B C}=\frac{a^{3} \cot \alpha}{24}=\frac{a^{3} \cot 30^{\circ}}{24}=\frac{a^{3} \sqrt{3}}{24}$

So choosing Answer D.

- $\quad$ Answer $A$ is wrong because of only substituting $\tan 30^{\circ}$.

Answer $B$ is wrong because of only replacing $\cos 30^{\circ}$.

- $\quad$ Answer $\mathrm{C}$ is wrong because students forget to divide 12 in the formula.

Question 10

$V_{S . A B C}=\frac{a^{3} \cot \alpha}{24}=\frac{(2 a)^{3} \cot 60^{\circ}}{24}=\frac{a^{3} \sqrt{3}}{9}$

Hence selecting Answer C.

- $\quad$ Answer A is wrong because of only substituting $2 a^{3}$.

- Answer B is wrong because students mistake $\cos 60^{\circ}$

- $\quad$ Answer $D$ is wrong because students mistake $\tan 60^{\circ}$.

Question 11

$V_{S . A B C D}=\frac{a^{2} \sqrt{4 b^{2}-2 a^{2}}}{6}=\frac{a^{2} \sqrt{4 \cdot(2 a)^{2}-2 a^{2}}}{6}=\frac{a^{3} \sqrt{14}}{6}$

Thus choosing Answer A.

- $\quad$ Answer B is wrong because students mistake "-" become "+".

- $\quad$ Answer $C$ is wrong because students substitute edge lengths with $a$.

- $\quad$ Answer D is wrong because of only replacing $2 a^{2}$.

Question 12

Volume of equilateral quadrilateral pyramid is $V_{S . A B C D}=\frac{a^{3} \sqrt{2}}{6}=\frac{(2 a)^{3} \sqrt{2}}{6}=\frac{a^{3} 4 \sqrt{2}}{3}$

Therefore selecting Answer B.

Answer $A$ is wrong because of only the original formula, has not replaced the length of the sides to the formula. 
- Answer $\mathrm{C}$ is wrong because students only substituting $2 a^{3}$.

- $\quad$ Answer $D$ is wrong because of only squared the length of the edge.

\section{Question 13}

$V_{S . A B C D}=\frac{a^{3} \tan \alpha}{6}=\frac{\left(\frac{a \sqrt{3}}{2}\right)^{3} \cdot \tan 45^{\circ}}{6}=\frac{a^{3} \sqrt{3}}{16}$

So choosing Answer C.

- Answer $A$ is wrong because wrong formula of equilateral triangular pyramid.

- $\quad$ Answer B is wrong because students used the wrong formula: $\frac{\sqrt{2} a^{3} \tan \beta}{6}$.

- $\quad$ Answer $D$ is wrong because students neglect to cube the length of edge.

\section{$\underline{\text { Question } 14}$}

$V_{S . A B C D}=\frac{a^{3} \tan \alpha}{6}=\frac{(2 a)^{3} \cdot \tan 60^{\circ}}{6}=\frac{a^{3} 4 \sqrt{3}}{3}$

Hence selecting Answer D.

- $\quad$ Answer $\mathrm{A}$ is wrong because of only substituting $2 a^{3}$.

- Answer $B$ is wrong because of the wrong formula: $\frac{\sqrt{2} a^{3} \tan \beta}{6}$.

- Answer $\mathrm{C}$ is wrong because students only replace the length of edge is $a$.

\section{$\underline{\text { Question } 15}$}

$V_{S . A B C D}=\frac{\sqrt{2} a^{3} \tan \beta}{6}=\frac{\sqrt{2} \cdot(a \sqrt{3})^{3} \cdot \tan 30^{\circ}}{6}=\frac{a^{3} \sqrt{2}}{2}$

Thus choosing Answer D.

- Answer $A$ is wrong because students only replace the length of edge is $a$.

- $\quad$ Answer B is wrong because students utilized of the wrong formula.

- Answer $\mathrm{C}$ is wrong because of the wrong formula: $\frac{a^{3} \tan \alpha}{6}$.
Question 16

$V_{S . A B C D}=\frac{\sqrt{2} a^{3} \tan \beta}{6}=\frac{\sqrt{2} \cdot(2 a)^{3} \cdot \tan 45^{\circ}}{6}=\frac{a^{3} 4 \sqrt{2}}{3}$

Therefore selecting Answer B.

- Answer $A$ is wrong because of not cube to the length of edge $2 a$.

- Answer $\mathrm{C}$ is wrong because students only replace the length of edge is $a$.

- $\quad$ Answer $D$ is wrong because students mistake $\cos 45^{\circ}$ or the wrong formula.

Question 17

$V_{S . A B C D}=\frac{a^{3} \sqrt{\tan ^{2} \gamma-1}}{6}=\frac{a^{3} \sqrt{\tan ^{2} 60^{\circ}-1}}{6}=\frac{a^{3} \sqrt{2}}{6}$

So, choosing Answer C.

- $\quad$ Answer $A$ is wrong because students neglect to square $\tan 60^{\circ}$.

- $\quad$ Answer B is wrong because students forget to divide 6 in the formula.

- $\quad$ Answer D is wrong because students mistake "-" become "+".

Question 18

$$
V_{S . A B C D}=\frac{4 b^{3} \tan \alpha}{3 \sqrt{\left(2+\tan ^{2} \alpha\right)^{3}}}=\frac{4 \cdot(2 a)^{3} \cdot \tan 45^{\circ}}{3 \sqrt{\left(2+\tan ^{2} 45^{\circ}\right)^{3}}}=\frac{a^{3} 32 \sqrt{3}}{27}
$$

Hence selecting Answer A.

- $\quad$ Answer B is wrong because students forget to cube $2 a$.

- $\quad$ Answer $\mathrm{C}$ is wrong because of only replace the length is $a$.

- $\quad$ Answer $D$ is wrong because students neglect to cube to expression in the root.

\section{Question 19}

$V_{S . A B C D}=\frac{4 b^{3} \tan \alpha}{3 \sqrt{\left(2+\tan ^{2} \alpha\right)^{3}}}=\frac{4 \cdot a^{3} \cdot \tan 60^{\circ}}{3 \sqrt{\left(2+\tan ^{2} 60^{\circ}\right)^{3}}}=\frac{a^{3} 4 \sqrt{15}}{75}$

Thus choosing Answer B.

- $\quad$ Answer $A$ is wrong because students forget to multiply 4 in the formula.

- $\quad$ Answer $C$ is wrong because students neglect to cube to expression in the root. 
- $\quad$ Answer $D$ is wrong because students forget to divide 3 in the formula.

\section{Question 20}

$$
V_{S . A B C}=\frac{S B^{3} \cdot \sin 2 \beta \cdot \tan \alpha}{12}=\frac{(a \sqrt{3})^{3} \cdot \sin \left(2 \cdot 30^{\circ}\right) \cdot \tan 45^{\circ}}{12}=\frac{3 a^{3}}{8} .
$$

Therefore selecting Answer A.

- $\quad$ Answer B is wrong because of only multiply $\sin 30^{\circ}$.

- $\quad$ Answer $\mathrm{C}$ is wrong because students mistake $\sin \left(2.45^{\circ}\right) \cdot \tan 30^{\circ}$.

- $\quad$ Answer $D$ is wrong because students forget to divide 12 in the formula.

\section{Question 21}

$V_{S . A B C}=\frac{S B^{3} \cdot \sin 2 \beta \cdot \tan \alpha}{12}=\frac{a^{3} \cdot \sin \left(2 \cdot 60^{\circ}\right) \cdot \tan 30^{\circ}}{12}=\frac{a^{3}}{24}$.

So choosing Answer C.

- Answer $A$ is wrong because students mistake $\sin 30^{\circ} \cdot \tan 60^{\circ}$.

- Answer B is wrong because students mistake $\sin \left(2.30^{\circ}\right) \cdot \tan 60^{\circ}$.

- $\quad$ Answer $D$ is wrong because students forget to divide 12 in the formula.

\section{Question 22}

$$
V_{S A B C}=\frac{a^{3} \sqrt{2}}{12}=\frac{(a \sqrt{2})^{3} \cdot \sqrt{2}}{12}=\frac{a^{3}}{3}
$$

Hence selecting Answer D.

- $\quad$ Answer A is wrong because students mistake in $a \sqrt{3}$.

- $\quad$ Answer B is wrong because of only square in $a \sqrt{2}$ or forget to multiply $\sqrt{2}$ in the formula.

- $\quad$ Answer $\mathrm{C}$ is wrong because students used the original formula, do not replace the edge length with $a \sqrt{2}$.

\section{Question 23}

$$
V_{S A B C}=\frac{a^{3} \sqrt{2}}{12}=\frac{(2 a)^{3} \cdot \sqrt{2}}{12}=\frac{2 \sqrt{2} a^{3}}{3}
$$

Thus choosing Answer B.

- $\quad$ Answer $A$ is wrong because of only substitute the length of edge is $a$.

- $\quad$ Answer $\mathrm{C}$ is wrong because students forget to cube $2 a$.

- $\quad$ Answer $D$ is wrong because students mistake in options.

Question 24

$$
\begin{aligned}
& V_{S . A B C}=\frac{\sqrt{2}}{12} \sqrt{\left(a^{2}+b^{2}-c^{2}\right)\left(b^{2}+c^{2}-a^{2}\right)\left(a^{2}+c^{2}-b^{2}\right)} \\
& =\frac{\sqrt{2}}{12} \sqrt{\left((6 a)^{2}+(5 a)^{2}-(4 a)^{2}\right)\left((5 a)^{2}+(4 a)^{2}-(6 a)^{2}\right)} \\
& =\frac{a^{3} 15 \sqrt{6}}{4}
\end{aligned}
$$

\section{Therefore selecting Answer A.}

- $\quad$ Answer B is wrong because students mistake "-“ become "+".

- $\quad$ Answer $\mathrm{C}$ is wrong because students forget to multiply $\frac{\sqrt{2}}{12}$ in the formula.

- $\quad$ Answer D is wrong because students mistake in the addition operation.

Question 25

$$
\begin{aligned}
& V_{S . A B C}=\frac{\sqrt{2}}{12} \sqrt{\left(a^{2}+b^{2}-c^{2}\right)\left(b^{2}+c^{2}-a^{2}\right)\left(a^{2}+c^{2}-b^{2}\right)} \\
& =\frac{\sqrt{2}}{12} \sqrt{\left(6^{2}+7^{2}-8^{2}\right)\left(7^{2}+8^{2}-6^{2}\right)\left(6^{2}+8^{2}-7^{2}\right)} \\
& =\frac{7 \sqrt{374}}{4} \mathrm{~cm}^{3}
\end{aligned}
$$

So choosing Answer C.

- $\quad$ Answer $A$ is wrong because students forget to multiply $\frac{\sqrt{2}}{12}$ in the formula.

- $\quad$ Answer B is wrong because students mistake "." become "+".

- $\quad$ Answer D is wrong because students neglect to take the square root.

Question 26

$$
V_{A B C D}=\frac{\sqrt{2 \cdot S_{1} S_{2} S_{3}}}{3}=\frac{\sqrt{2 \cdot 15 \cdot 20 \cdot 18}}{3}=20 \sqrt{3} \mathrm{~cm}^{3} .
$$


Hence selecting Answer D.

- $\quad$ Answer $A$ is wrong because students neglect to divide 3 in the formula.

- $\quad$ Answer B is wrong because students forget to multiply 2 in the root.

- $\quad$ Answer $\mathrm{C}$ is wrong because students mistake in $\frac{a b c}{6}$

\section{Question 27}

$V_{A B C D}=\frac{\sqrt{2 \cdot S_{1} S_{2} S_{3}}}{3}=\frac{\sqrt{2 \cdot a^{2} \cdot 2 a^{2} \cdot 3 a^{2}}}{3}=\frac{2 \sqrt{3}}{3} a^{3}$.

Thus choosing Answer A.

- $\quad$ Answer B is wrong because students neglect to divide 3 in the formula.

- $\quad$ Answer $\mathrm{C}$ is wrong because students forget to multiply 2 in the root.

- $\quad$ Answer $\mathrm{D}$ is wrong because mistake in $\frac{a b c}{6}$.

\section{$\underline{\text { Question } 28}$}

$V_{S . A B C}=\frac{a b c}{6}=\frac{5.10 .20}{6}=\frac{500}{3} \mathrm{~cm}^{3}$

Therefore selecting Answer B.

- $\quad$ Answer $A$ is wrong because students forget to change $\mathrm{cm}$.

- Answer $\mathrm{C}$ is wrong because of mistake in $\frac{\sqrt{2 \cdot S_{1} S_{2} S_{3}}}{3}$.

- $\quad$ Answer D is wrong because students mistake "." become "+".

\section{Question 29}

$V_{S . A B C}=\frac{a b c}{6}=\frac{3 a \cdot 2 a \cdot a}{6}=a^{3}$

So choosing Answer C.

- $\quad$ Answer $A$ is wrong because students forget to divide 6 in the formula.

- $\quad$ Answer B is wrong because students mistake "." become "+".

- Answer D is wrong because of mistaking in $\frac{\sqrt{2 \cdot S_{1} S_{2} S_{3}}}{3}$.
Question 30

$V_{S . A B C}=\frac{a b c}{6} \sqrt{1-\cos ^{2} \alpha-\cos ^{2} \beta-\cos ^{2} \gamma+2 \cos \alpha \cos \beta \cos \gamma}$

$=\frac{a \cdot a \cdot a}{6} \sqrt{1-\cos ^{2} 60^{\circ}-\cos ^{2} 60^{\circ}-\cos ^{2} 90^{\circ}+2 \cos 60^{\circ} \cos 60^{\circ} \cos 90^{\circ}}$

$=\frac{a^{3} \sqrt{2}}{12}$

Hence selecting Answer B.

- $\quad$ Answer $A$ is wrong because students neglect to cube $\cos \alpha$ and $\cos \beta$.

- $\quad$ Answer $\mathrm{C}$ is wrong because students mistake "-“ become "+".

- $\quad$ Answer $D$ is wrong because students forget to take the square root.

Question 31

$$
V_{S . A B C}=\frac{a b c}{6} \sqrt{1-\cos ^{2} \alpha-\cos ^{2} \beta-\cos ^{2} \gamma+2 \cos \alpha \cos \beta \cos \gamma}
$$

$=\frac{a \cdot a \cdot 2 a}{6} \sqrt{\begin{array}{l}1-\cos ^{2} 60^{\circ}-\cos ^{2} 90^{\circ}-\cos ^{2} 120^{\circ} \\ +2 \cos 60^{\circ} \cos 90^{\circ} \cos 120^{\circ}\end{array}}$

$=\frac{a^{3} \sqrt{2}}{6}$

Thus choosing Answer C.

- $\quad$ Answer A is wrong because students mistake "-“ become "+".

- $\quad$ Answer $B$ is wrong because students neglect to cube $\cos \alpha$ and $\cos \beta$.

- $\quad$ Answer $D$ is wrong because students forget to take the square root.

Question 32

$V_{S \cdot A B C}=\frac{1}{6} a b d \sin \alpha=\frac{1}{6} \cdot a \cdot a \sqrt{3} \cdot 8 a \cdot \sin 60^{\circ}=2 a^{3}$

Therefore selecting Answer A.

- $\quad$ Answer B is wrong because of mistaking $\sin 60^{\circ}$ to $\cos 60^{\circ}$.

- $\quad$ Answer $\mathrm{C}$ is wrong because students neglect to divide 6 in the formula.

- $\quad$ Answer D is wrong because students forget to multiply to $d$. 


\section{Question 33}

$V_{S \cdot A B C}=\frac{1}{6} a b d \sin \alpha=\frac{1}{6} \cdot 3 a \cdot 3 a \cdot 2 a \cdot \sin 90^{\circ}=3 a^{3}$

So choosing Answer D.

- $\quad$ Answer A is wrong because students forget to mutltiply lack of an edge $3 a$.

- $\quad$ Answer B is wrong because students neglect to multiply to $d$.

- $\quad$ Answer $\mathrm{C}$ is wrong because students forget to divide 6 in the formula.

\section{Question 34}

$V_{S . A B C}=\frac{2 S_{1} S_{2} \sin \alpha}{3 a}=\frac{2 \cdot 2 a^{2} \cdot 5 a^{2} \cdot \sin 30^{\circ}}{3 \cdot 2 a}=\frac{5}{3} a^{3}$

Hence selecting Answer B.

- $\quad$ Answer $\mathrm{A}$ is wrong because students neglect to divide 3 in the formula.

- $\quad$ Answer $C$ is wrong because students forget to multiply 2 in the formula.

- Answer $D$ is wrong because of missing in $S A=a$

\section{$\underline{\text { Question } 35}$}

$V_{S . A B C}=\frac{2 S_{1} S_{2} \sin \alpha}{3 a}=\frac{2 \cdot \frac{\sqrt{6}}{2} a^{2} \cdot \frac{\sqrt{3}}{2} a^{2} \cdot \sin 60^{\circ}}{3 \cdot a \sqrt{2}}=\frac{\sqrt{3}}{4} a^{3}$

Thus choosing Answer A.

- $\quad$ Answer $B$ is wrong students neglect to divide 3 in the formula.

- Answer $\mathrm{C}$ is wrong because of mistaking the length of edge is $a$.

- $\quad$ Answer $D$ is wrong because students forget to multiply 2 in the formula.

\section{Question 36}

$V_{S . A B C}=\frac{a b c}{6} \sin \alpha \sin \beta \sin \gamma=\frac{2 a \cdot 3 a \cdot 5 a}{6} \cdot \sin 30^{\circ} \cdot \sin 15^{\circ} \cdot \sin 45^{\circ}$

$=\frac{-5+5 \sqrt{3}}{8} a^{3}$

Therefore selecting Answer B.

Answer $A$ is wrong because students forget to divide 6 in the formula.
Answer $C$ is wrong because students mistake the product of 3 edges to the sum of 3 edges.

Answer $D$ is wrong because students mistake sin to cos in the formula.

\section{Question 37}

$$
\begin{aligned}
& V_{S . A B C}=\frac{a b c}{6} \sin \alpha \sin \beta \sin \gamma=\frac{a \cdot a \sqrt{2} \cdot a \sqrt{3}}{6} \cdot \sin 60^{\circ} \\
& \cdot \sin 30^{\circ} \cdot \sin 45^{\circ}=\frac{a^{3}}{8}
\end{aligned}
$$

So choosing Answer B.

- $\quad$ Answer $A$ is wrong because students forget to divide 6 in the formula.

- Answer $C$ is wrong because of mistaking the length of edge is $a$.

- Answer D is wrong because of missing in options.

\section{Question 38}

$V=\frac{a^{3}}{6}=\frac{(2 a)^{3}}{6}=\frac{4 a^{3}}{3}$

Hence selecting Answer A.

- $\quad$ Answer B is wrong because students forget to cube the length of edge.

- Answer $\mathrm{C}$ is wrong because students only square the length of edge.

- Answer $D$ is wrong because students only replace the length of edge is $a$.

\section{Question 39}

Tỷ số $\frac{a^{3}}{V}=\frac{a^{3}}{\frac{2 a^{3} \sqrt{2}}{27}}=\frac{27 \sqrt{2}}{4}$

Thus choosing Answer D.

- Answer $A$ is wrong because of missing in the formula.

- $\quad$ Answer B is wrong because of mistaking in the formula and the score of $\frac{a^{3}}{V}$.

- $\quad$ Answer $\mathrm{C}$ is wrong because students neglect to take the score of $\frac{a^{3}}{V}$, only calculating the volume. 


\section{$\underline{\text { Question } 40}$}

$$
V=\frac{a^{3}(15+7 \sqrt{5})}{4}=\frac{(2 a)^{3}(15+7 \sqrt{5})}{4}=(30+14 \sqrt{5}) a^{3}
$$

Therefore selecting Answer C.

- Answer A is wrong because students forget to cube the length of edge.

- Answer B is wrong because of only square the length of edge.

- Answer $D$ is wrong because students only replace the length of edge is $a$.

We now turn on the posteriori analysis in the next sub-section.

\subsection{Posterior Analysis}

\subsubsection{Introducing IATA Question Analysis Software}

We execute IATA software to analyze the above results. This software can be downloaded via the link: https://polymetrika.com/Downloads/IATA.

\subsubsection{Experimental Results of Approach 1}

Experimental topics include 40 questions given to 120 students from Nguyen Viet Hong High School, Chau Van Liem High School, Phan Van Tri High School, Ly Tu Trong High School for the Gifted, Pedagogical Practice High School, Phan Ngoc Hien High School, An Khanh High School, Nga Sau High School, High School Bui Huu Nghia, Luu Huu Phuoc High School to do the test with 90 minutes. The results obtained from IATA software are given in Table $\mathbf{2}$, where:

Questions without issues,

Questions to consider,

Questions have issues.

Discr is discriminant; Pval is difficulty; PBis is correlation coefficient according to classical test theory. The coefficients $a$ and $b$ are discriminants, the difficulty according to the test theory answers the IRT question. The results of the IATA show that the questions that are well used are 14 questions, which can be used as 23 questions and 3 questions have issues are questions 20, 21, and 27. Therefore, 3 questions have issues are excluded to proceed to approach 2.

\subsubsection{Experimental Results of Approach 2}

From 40 exercises of group A students drawn from approach 1 and 37 exercises of group B students from Ly Tu Trong High School for the Gifted, High School
Pedagogical Practice, Chau Van Liem High School, Bui Huu Nghia High School, Nguyen Viet Hong High School are analyzed as follows:

\subsubsection{Evaluation of Experimental Results}

Based on Tables $\mathbf{2}$ and $\mathbf{3}$, the control group has an average score of $100 \%$ in good mathematics, but the correct rate of sentences is low, for example: questions 34 and 35 have a rate of $21.62 \%$; questions 37 and 39 have a rate of $27.03 \%$; and question 40 has a rate of $35.14 \%$.

Meanwhile, the experimental group had a lower mathematics score than the control group, but the rate of correct answers was higher, most of them were over $80 \%$, the lowest rate was 28 with $75 \%$. This shows the effectiveness of using quick calculation formulas.

Through the experimental process, the results showed that: the experimental purpose has been completed, and the feasibility and effectiveness of using the formulas for calculating the volume of the polyhedron in spatial geometry has been affirmed. Implementing geometric exercises with quick calculation formulas is a tool to support students to receive, understand and apply in appropriate situations, thereby helping students to achieve good results in exams.

\section{CONCLUDING REMARKS AND INFERENCE}

As stated in the Introduction, in the age of globalization, an effective leadership skill is the ability for quick calculation of work-related problems. From an economic perspective, fast computation often gives a competitive advantage in business, where speed, efficiency and accuracy are essential. Quick calculation techniques are a central problem in modern mathematics because it shortens the time for solving technical problems.

This paper has developed convenient and straightforward formulas to calculate quickly the polyhedron volume in spatial geometry. The empirical analysis was conducted at Can Tho City to evaluate efficiently the proposed formulas. This paper provides a foundation for teachers and students to approach the method of quick solving spatial geometry exercises using simple formulas. The experimental results show that students can solve exercises faster with the help of quick calculation formulas. This is a useful tool that can help students be more confident in the face of problems of calculating volumes in space geometry, which can be useful for commercial purposes. 
Table 2: Difficulty, Discriminant and Property of Sentences in the Test

\begin{tabular}{|c|c|c|c|c|c|c|c|}
\hline Use & Name & Discr & PVal & PBis & a & $b$ & c \\
\hline$\square$ & $Q_{1}$ & 0.00 & 1.00 & $\mathrm{NaN}$ & $-1,00$ & $-999,00$ & 0.00 \\
\hline$\square$ & Q2 & 0.00 & 1.00 & $\mathrm{NaN}$ & $-1,00$ & $-999,00$ & 0.00 \\
\hline$\square$ & Q3 & 0,50 & 0,46 & 0.51 & 0.73 & 0.16 & 0.00 \\
\hline$\square$ & Q4 & 0.25 & 0.31 & 0.21 & 0,27 & 1.85 & 0.00 \\
\hline$\nabla$ & $Q 5$ & 0.00 & 1.00 & $\mathrm{NaN}$ & $-1,00$ & $-999,00$ & 0.00 \\
\hline$\square$ & Q6 & 0,00 & 1.00 & $\mathrm{NaN}$ & -1.00 & $-999,00$ & 0.00 \\
\hline$\square$ & Q7 & $-0,25$ & 0.77 & -0.22 & $-1,00$ & $-999,00$ & 0.00 \\
\hline$\square$ & Q8 & 0.00 & 1,00 & $\mathrm{NaN}$ & $-1,00$ & $-999,00$ & 0.00 \\
\hline$\square$ & Q9 & 0.50 & 0.38 & 0.47 & 0.67 & 0,51 & 0.00 \\
\hline$\square$ & Q10 & 1.00 & 0.62 & 0.71 & 1.43 & -0.34 & 0.00 \\
\hline$\square$ & Q11 & 0,00 & 0.92 & -0.01 & $-1,00$ & $-999,00$ & 0,00 \\
\hline$\square$ & Q12 & 0.00 & 1.00 & $\mathrm{NaN}$ & -1.00 & -999.00 & 0.00 \\
\hline$\nabla$ & Q13 & 0.67 & 0.77 & 0.78 & 2,18 & $-0,77$ & 0.00 \\
\hline$\square$ & Q14 & 1.00 & 0.69 & 0.80 & 2,13 & -0.52 & 0.00 \\
\hline$\square$ & Q15 & 0.00 & 1.00 & $\mathrm{NaN}$ & $-1,00$ & $-999,00$ & 0.00 \\
\hline$\square$ & Q16 & 0.00 & 0.92 & -0.09 & $-1,00$ & $-999,00$ & 0,00 \\
\hline$\square$ & Q17 & 0.00 & 1.00 & $\mathrm{NaN}$ & $-1,00$ & $-999,00$ & 0.00 \\
\hline$\nabla$ & Q18 & 0,33 & 0.92 & 0.46 & 1,21 & $-1,87$ & 0.00 \\
\hline$\square$ & Q19 & 0.67 & 0.85 & 0.92 & 5.18 & $-0,98$ & 0.00 \\
\hline$\square$ & $\Delta \quad$ Q20 & 0.08 & 0.69 & 0.25 & 0.32 & $-1,59$ & 0.00 \\
\hline
\end{tabular}

\begin{tabular}{|c|c|c|c|c|c|c|c|}
\hline Use & Name & Discr & PVal & PBis & a & $\mathrm{b}$ & c \\
\hline$\square$ & $\Delta \mathrm{Q} 21$ & 0.08 & 0.54 & 0.17 & 0.20 & -0.46 & 0.00 \\
\hline$\square$ & Q22 & -0.25 & 0.92 & -0.17 & -1.00 & -999.00 & 0.00 \\
\hline$\nabla$ & $>Q 23$ & 0.00 & 1.00 & $\mathrm{NaN}$ & $-1,00$ & $-999,00$ & 0.00 \\
\hline$\square$ & Q24 & 0.42 & 0.69 & 0,38 & 0.54 & $-1,03$ & 0.00 \\
\hline$\square$ & Q25 & 0.67 & 0.69 & 0.66 & 1,28 & $-0,62$ & 0.00 \\
\hline$\square$ & Q26 & 0.00 & 0.85 & $-0,08$ & $-1,00$ & $-999,00$ & 0.00 \\
\hline$\square$ & Q27 & 0.17 & 0.54 & 0.09 & 0.10 & -0.91 & 0.00 \\
\hline$\square$ & Q28 & 0.00 & 0.85 & -0.08 & $-1,00$ & $-999,00$ & 0.00 \\
\hline$\square$ & Q29 & 0.00 & 1,00 & $\mathrm{NaN}$ & $-1,00$ & $-999,00$ & 0.00 \\
\hline$\square$ & Q30 & 0.67 & 0.85 & 0.92 & 5.18 & -0.98 & 0.00 \\
\hline$\square$ & Q31 & 0.25 & 0.23 & 0.27 & 0.39 & 1,96 & 0.00 \\
\hline$\square$ & Q32 & 0.42 & 0.54 & 0.13 & 0.15 & -0.61 & 0.00 \\
\hline$\square$ & Q33 & 0.67 & 0,62 & 0.49 & 0.71 & -0.49 & 0.00 \\
\hline$\square$ & Q34 & 0.42 & 0,38 & 0.34 & 0.44 & 0.71 & 0.00 \\
\hline$\square$ & Q35 & 0.17 & 0.31 & 0,26 & 0.33 & 1.52 & 0.00 \\
\hline$\square$ & Q36 & 0.25 & 0.31 & 0.17 & 0.21 & 2,36 & 0.00 \\
\hline$\square$ & Q37 & -0.33 & 0.15 & $-0,28$ & $-1,00$ & -999.00 & 0.00 \\
\hline$\nabla$ & Q38 & 0.67 & 0.85 & 0.92 & 5.18 & -0.98 & 0.00 \\
\hline$\nabla$ & Q39 & 0.17 & 0.46 & 0.34 & 0.42 & 0.24 & 0.00 \\
\hline$\square$ & $>Q 40$ & 0,33 & 0.77 & 0.43 & 0.68 & $-1,28$ & 0.00 \\
\hline
\end{tabular}


Table 3: The Rate of Correct Answers to each Question of the Control Group and Experimental Group

\begin{tabular}{|c|c|c|c|c|c|}
\hline Question & $\begin{array}{l}\text { Control group } \\
\text { (Group A) }\end{array}$ & $\begin{array}{l}\text { Experimental group } \\
\text { (Group B) }\end{array}$ & Question & $\begin{array}{c}\text { Control group } \\
\text { (Group A) }\end{array}$ & $\begin{array}{c}\text { Experimental group } \\
\text { (Group B) }\end{array}$ \\
\hline 1 & $100 \%$ & $100 \%$ & 22 & $97.30 \%$ & $95 \%$ \\
\hline 3 & $97.30 \%$ & $97.50 \%$ & 24 & $45.95 \%$ & $97.50 \%$ \\
\hline 4 & $81.08 \%$ & $97.50 \%$ & 25 & $56.76 \%$ & $100 \%$ \\
\hline 6 & $97.30 \%$ & $95 \%$ & 28 & $78.38 \%$ & $75 \%$ \\
\hline 7 & $97.30 \%$ & $95 \%$ & 29 & $100 \%$ & $97.50 \%$ \\
\hline 8 & $94.59 \%$ & $95 \%$ & 30 & $86.49 \%$ & $100 \%$ \\
\hline 9 & $48.65 \%$ & $97.50 \%$ & 31 & $37.84 \%$ & $97.50 \%$ \\
\hline 13 & $97.30 \%$ & $97.50 \%$ & 35 & $21.62 \%$ & $97.50 \%$ \\
\hline 14 & $100 \%$ & $92.50 \%$ & 36 & $37.84 \%$ & $97.50 \%$ \\
\hline 15 & $78.38 \%$ & $95 \%$ & 37 & $27.03 \%$ & $97.50 \%$ \\
\hline 16 & $83.78 \%$ & $95 \%$ & 38 & $67.56 \%$ & $97.50 \%$ \\
\hline 17 & $91.89 \%$ & $100 \%$ & 39 & $27.03 \%$ & $82.50 \%$ \\
\hline 18 & $100 \%$ & $90 \%$ & 40 & $35.14 \%$ & $85 \%$ \\
\hline 19 & $83.78 \%$ & $87.50 \%$ & & & \\
\hline
\end{tabular}

Moreover, the formulas for quick calculation of the polyhedron volume in spatial geometry contributed importantly and significantly to the solution of multiple choice tests. This is also a premise to develop simple and faster calculation formulas in solving problems of spatial geometry and a tool for research in developing cognate topics. As an extension of the paper, we can directly use software such as R, GeoGebra for programming in learning and teaching. This is a topic of ongoing research.

\section{ACKNOWLEDGEMENT}

The authors wish to thank a referee for very helpful comments and suggestions. For financial support, the second author is most grateful to the Australian Research Council and the Ministry of Science and Technology (MOST), Taiwan.

\section{REFERENCES}

Azevedo, J.M. (2015), E-assessment in mathematics courses with multiple-choice questions tests. In 7th International Conference on Computer Supported Education (pp. 260266). Copyright 2015 SCITEPRESS (Science and Technology Publications, Lda.). https://doi.org/10.5220/0005452702600266
Bakó, M. (2003), Different projecting methods in teaching spatial geometry. In Proceedings of the Third Conference of the European society for Research in Mathematics Education.

Bertoline, G.R. (1991), Using 3D geometric models to teach spatial geometry concepts. Engineering Design Graphics Journal, 55(1), 37-47.

Boslaugh, S. (2013), Statistics in a Nutshell: A Desktop Quick Reference (second edition). California (USA): O'Reilly Media.

Bribena, K. (2019), Globalization and knowledge production in the world: A critical analysis of the place of Africa. Journal of Reviews on Global Economics, 8, 12-20. https://doi.org/10.6000/1929-7092.2019.08.02

Chazan, D. (2000), Beyond Formulas in Mathematics and Teaching: Dynamics of the High School Algebra Classroom. New York: Teachers College Press.

Grabinski, H., Konrad, B., Nordholz, P. (2002), Simple formulas to calculate the line parameters of interconnects on conducting substrates. IEEE 7th Topical Meeting on Electrical Performance of Electronic Packaging.

Jones, K. (2002), Issues in the Teaching and Learning of Geometry. In: Linda Haggarty (Ed), Aspects of Teaching Secondary Mathematics: perspectives on practice. London: RoutledgeFalmer. Chapter 8, pp. 121-139.

Kosa, T., and Karakus, F. (2010), Using dynamic geometry software Cabri 3D for teaching analytic geometry. Procedia-Social and Behavioral Sciences, 2(2), 1385-1389. https://doi.org/10.1016/j.sbspro.2010.03.204

Kurtulus, A., and Uygan, C. (2010), The effects of Google Sketchup based geometry activities and projects on spatial visualization ability of student mathematics teachers. Procedia-Social and Behavioral Sciences, 9, 384-389. https://doi.org/10.1016/j.sbspro.2010.12.169 
Laborde, C., Kynigos, C., Hollebrands, K., and Strässer, R. (2006), Teaching and learning geometry with technology. In Handbook of research on the psychology of mathematics education (pp. 275-304). Brill Sense. https://doi.org/10.1163/9789087901127_011

Lesage, E., Valcke, M., and Sabbe, E. (2013), Scoring methods for multiple choice assessment in higher education-Is it still a matter of number right scoring or negative marking?. Studies in Educational Evaluation, 39(3), 188-193. https://doi.org/10.1016/j.stueduc.2013.07.001

Lindberg, S. M., Hyde, J. S., Petersen, J. L., and Linn, M. C. (2010), New trends in gender and mathematics performance: a meta-analysis. Psychological bulletin, 136(6), 1123. https://doi.org/10.1037/a0021276

Lindner, M. A., Eitel, A., Thoma, G. B., Dalehefte, I. M., Ihme, J. M., and Köller, O. (2014), Tracking the decision-making process in multiple-choice assessment: Evidence from eye movements. Applied Cognitive Psychology, 28(5), 738-752. https://doi.org/10.1002/acp.3060
Little, J. L., Bjork, E. L., Bjork, R. A., and Angello, G. (2012), Multiplechoice tests exonerated, at least of some charges: Fostering test-induced learning and avoiding test-induced forgetting. Psychological science, 23(11), 1337-1344. https://doi.org/10.1177/0956797612443370

Moss, J., Hawes, Z., Naqvi, S., and Caswell, B. (2015), Adapting Japanese Lesson Study to enhance the teaching and learning of geometry and spatial reasoning in early years classrooms: a case study. Zdm, 47(3), 377-390. https://doi.org/10.1007/s11858-015-0679-2

Sousa M. J., Santos V., Sacavem A., Reis I.P., and Sampaio M.C. (2019), 4.0 Leadership skills in hospitality sector. Journal of Reviews on Global Economics, 8, 105-117. https://doi.org/10.6000/1929-7092.2019.08.11

Torres, C., Lopes, A.P., Babo, L., and Azevedo, J. (2011), Improving Multiple-Choice Questions. Online Submission.

Wheatley, G.H. (2007), Quick Draw: Developing Spatial Sense in Mathematics. Florida State University.

DOI: https://doi.org/10.6000/1929-7092.2019.08.71

(C) 2019 Tuan et al.; Licensee Lifescience Global.

This is an open access article licensed under the terms of the Creative Commons Attribution Non-Commercial License (http://creativecommons.org/licenses/by-nc/3.0/) which permits unrestricted, non-commercial use, distribution and reproduction in any medium, provided the work is properly cited. 